\title{
Equivalent Circuits Applied in Electrochemical Impedance Spectroscopy and Fractional Derivatives with and without Singular Kernel
}

\author{
J. F. Gómez-Aguilar, ${ }^{1}$ J. E. Escalante-Martínez, ${ }^{2}$ C. Calderón-Ramón, ${ }^{2}$ \\ L. J. Morales-Mendoza, ${ }^{3}$ M. Benavidez-Cruz, ${ }^{2}$ and M. Gonzalez-Lee ${ }^{3}$ \\ ${ }^{1}$ CONACYT-Centro Nacional de Investigación y Desarrollo Tecnológico, Tecnológico Nacional de México, \\ Interior Internado Palmira S/N, Colonia Palmira, 62490 Cuernavaca, MOR, Mexico \\ ${ }^{2}$ Facultad de Ingeniería Mecánica y Eléctrica, Universidad Veracruzana, Avenida Venustiano Carranza S/N, \\ Colonia Revolución, 93390 Poza Rica, VER, Mexico \\ ${ }^{3}$ Facultad de Ingeniería Electrónica y Comunicaciones, Universidad Veracruzana, Avenida Venustiano \\ Carranza S/N, Colonia Revolución, 93390 Poza Rica, VER, Mexico
}

Correspondence should be addressed to J. F. Gómez-Aguilar; jgomez@ier.unam.mx

Received 2 February 2016; Accepted 26 April 2016

Academic Editor: Alexander Iomin

Copyright (C) 2016 J. F. Gómez-Aguilar et al. This is an open access article distributed under the Creative Commons Attribution License, which permits unrestricted use, distribution, and reproduction in any medium, provided the original work is properly cited.

We present an alternative representation of integer and fractional electrical elements in the Laplace domain for modeling electrochemical systems represented by equivalent electrical circuits. The fractional derivatives considered are of Caputo and Caputo-Fabrizio type. This representation includes distributed elements of the Cole model type. In addition to maintaining consistency in adjusted electrical parameters, a detailed methodology is proposed to build the equivalent circuits. Illustrative examples are given and the Nyquist and Bode graphs are obtained from the numerical simulation of the corresponding transfer functions using arbitrary electrical parameters in order to illustrate the methodology. The advantage of our representation appears according to the comparison between our model and models presented in the paper, which are not physically acceptable due to the dimensional incompatibility. The Markovian nature of the models is recovered when the order of the fractional derivatives is equal to 1 .

\section{Introduction}

Electrochemical Impedance Spectroscopy (EIS) is widely used to investigate the interfacial and bulk properties of materials, interfaces of electrode-electrolyte, and the interpretation of phenomena such as electrocatalysis, corrosion, or behavior of coatings on metallic substrates. This technique relates directly measurements of impedance and phase angle as functions of frequency, voltage, or current applied. The stimulus is an alternating current signal of low amplitude intended to measure the electric field or potential difference generated between different parts of the sample. The relationship between the data of the applied stimulus and the response obtained as a function of frequency provides the impedance spectrum of samples studied [1]. Transfer function analysis is a mathematical approach to relate an input signal (or excitation) and the system's response. The ratio formed by the pattern of the output and the input signal makes it possible to find the zeros and poles, respectively. To analyze the behavior of the transfer function in the frequency domain, several graphical methods were used, such as Bode plots that provide a graphical representation of the magnitude and phase versus frequency of the transfer function and the Nyquist diagrams that are polar plots of impedance modulus and phase lag. It is very common in the literature to analyze impedance results by a physical model; this model is expressed by a mathematical representation and usually is represented by equivalent electrical circuits composed of 
elements such as resistors, capacitors, inductors, the Constant Phase Element (CPE), and Warburg elements (which is a special case of the $\mathrm{CPE}$ ). Although the resulting model is not necessarily unique, it describes the system with great precision in the range of frequencies studied [1].

Fractional calculus (FC) is the investigation and treatment of mathematical models in terms of derivatives and integrals of arbitrary order [2-5]. In the last years, the interest in the field has considerably increased due to many practical potential applications [6-13]. In the literature, a number of definitions of the fractional derivatives have been introduced, namely, the Hadamard, Erdelyi-Kober, RiemannLiouville, Riesz, Weyl, Grünwald-Letnikov, Jumarie, and the Caputo representation [2-5]. For example, for the Caputo representation, the initial conditions are expressed in terms of integer-order derivatives having direct physical significance [14], this definition is mainly used to include memory effects. Recently, Caputo and Fabrizio in [15] present a new definition of fractional derivative without a singular kernel; this derivative possesses very interesting properties, for instance, the possibility to describe fluctuations and structures with different scales. Furthermore, this definition allows for the description of mechanical properties related to damage, fatigue, and material heterogeneities. Properties of this new fractional derivative are reviewed in detail in [16].

Measurements of properties of materials, interfaces of electrode-electrolyte, corrosion, tissue properties of protein fibers, semiconductors and solid-state devices, fuel cells, sensors, batteries, electrochemical capacitors, coatings, and electrochromic materials have shown that their impedance behavior can only be modeled by using Warburg elements in conjunction with resistors, dispersive inductors, or CPE elements [17]. In this context, FC allows the investigation of the nonlocal response of electrochemical systems, this being the main advantage when compared with classical calculus. Some researches concerning EIS introduce FC; for example, the authors of [18] study the electrical impedance of vegetables and fruits from a FC perspective; the experiments are developed for measuring the impedance of botanical elements and the results are analyzed using Bode and Nyquist diagrams. In [19] a technique is presented to extract the parameters that characterize a dispersion Cole-Cole impedance model. Oldham in [20] describes how fractional differential equations have influenced the electrochemistry. Other applications of fractional calculus in electrochemical impedance are given in [21-26].

Unlike the work of the authors mentioned above, in which the pass from an ordinary derivative to a fractional one is direct, Gómez-Aguilar et al. in [12] analyze the ordinary derivative operator and try to bring it to the fractional form in a consistent manner. Following this idea we present an alternative representation of integer and fractional electrical elements in the Laplace domain for modeling electrochemical systems represented by equivalent electrical circuits; the order of the fractional equation is $0<\gamma, \beta \leq 1$. In this representation an auxiliary parameter $\alpha$ is introduced; this parameter characterizes the existence of the fractional temporal components and relates the time constant of the system.
The paper is organized as follows: Section 2 explains the basic concepts of the FC, Section 3 presents the examples considered and the interpretation of typical diagrams, and the conclusions are given in Section 4.

\section{Introduction to Fractional Calculus}

The use of Caputo Fractional Derivative (CD) in Physics is gaining importance because of the specific properties: the derivative of a constant is zero and the initial conditions for the fractional order differential equations can be given in the same manner as for the ordinary differential equations with a known physical interpretation [4].

The CD is defined as follows [4]:

$$
{ }_{0}^{C} D_{t}^{\gamma} f(t)=\frac{1}{\Gamma(n-\gamma)} \int_{0}^{t} \frac{f^{(n)}(\alpha)}{(t-\alpha)^{\gamma-n+1}} d \alpha
$$

where $d^{\varphi} / d t^{\varphi}={ }_{a}^{C} D_{t}^{\varphi}$ is a CD with respect to $t, \varphi \in R$ is the order of the fractional derivative, and $\Gamma(\cdot)$ represents the gamma function. [4]:

The Laplace transform of the CD has the following form

$$
L\left[{ }_{0}^{C} D_{t}^{\gamma} f(t)\right]=S^{\gamma} F(S)-\sum_{k=0}^{m-1} S^{\gamma-k-1} f^{(k)}(0) .
$$

The Caputo-Fabrizio fractional derivative (CF) is defined as follows $[15,16]$ :

$$
{ }_{0}^{\mathrm{CF}} \mathscr{D}_{t}^{\gamma} f(t)=\frac{M(\gamma)}{1-\gamma} \int_{0}^{t} \dot{f}(\alpha) \exp \left[-\frac{\gamma(t-\alpha)}{1-\gamma}\right] d \alpha,
$$

where $d^{\gamma} / d t^{\gamma}={ }_{0}^{\mathrm{CF}} D_{t}^{\gamma}$ is a CF with respect to $t$ and $M(\gamma)$ is a normalization function such that $M(0)=M(1)=1$; in this definition the derivative of a constant is equal to zero, but, unlike the usual Caputo definition (1), the kernel does not have a singularity at $t=\alpha$.

If $n \geq 1$ and $\gamma \in[0,1]$, the CF fractional derivative, ${ }_{0}^{\mathrm{CF}} \mathscr{D}_{t}^{(\gamma+n)} f(t)$, of order $(n+\gamma)$, is defined by

$$
{ }_{0}^{\mathrm{CF}} \mathscr{D}_{t}^{(\gamma+n)} f(t)={ }_{0}^{\mathrm{CF}} \mathscr{D}_{t}^{(\gamma)}\left({ }_{0}^{\mathrm{CF}} \mathscr{D}_{t}^{(n)} f(t)\right) .
$$

The Laplace transform of (3) is defined as follows $[15,16]$ :

$$
\begin{aligned}
L & {\left[{ }_{0}^{\mathrm{CF}} \mathscr{D}_{t}^{(\gamma+n)} f(t)\right] } \\
& =\frac{1}{1-\gamma} L\left[f^{(\gamma+n)} t\right] L\left[\exp \left(-\frac{\gamma}{1-\gamma} t\right)\right] \\
& =\frac{s^{n+1} L[f(t)]-s^{n} f(0)-s^{n-1} f^{\prime}(0) \cdots-f^{(n)}(0)}{s+\gamma(1-s)} .
\end{aligned}
$$

For this representation in the time domain it is suitable to use the Laplace transform $[15,16]$. 
From this expression we have

$$
\begin{aligned}
L\left[{ }_{0}^{\mathrm{CF}} \mathscr{D}_{t}^{\gamma} f(t)\right] & =\frac{s L[f(t)]-f(0)}{s+\gamma(1-s)}, \quad n=0, \\
L\left[{ }_{0}^{\mathrm{CF}}{ }_{t}^{(\gamma+1)} f(t)\right] & =\frac{s^{2} L[f(t)]-s f(0)-\dot{f}(0)}{s+\gamma(1-s)},
\end{aligned}
$$

\section{Electrochemical Impedance and the Interpretation of Typical Diagrams}

Regarding the equivalent circuits, there is a diversity of models used. The most common is to adjust the system to a simple model or one that includes a bilayer structure, either with RC groups in parallel or in series model, although there are cases where it is appropriate to include the Warburg impedance element to consider possible diffusive processes on the surface. Generally, using a complex equivalent circuit is not necessary to obtain a good characterization of the real system; commonly a simple electrical circuit is the first choice and increases its complexity when knowledge of the electrochemical behavior of the system also increases.

The Cole impedance model is based on replacing the ideal capacitor in the Debye model $[27,28]$. Cole model is represented by a series resistor $R_{s}$, a capacitor $C_{p}$, and a resistor in parallel $R_{p}$. In general it reflects the electrical resistance of the interface sample-electrode and maintains a negligible value with respect to $R_{p} ; \gamma$ is the order of the power that best fits the model obtained, $\gamma \in(0 ; 1)$, giving an ideal capacitor when it is 1 . Using the algebraic representation of the circuit can be said to represent the total impedance as

$$
Z_{T}(s)=R_{s}+\frac{R_{p}}{1+\left(s R_{p} C_{p}\right)^{\gamma}},
$$

where $s=j \omega$.

On electric structures RC type, bias resistor $R$ represents the charge transfer resistance and capacitance $C$ of double layer; the CPE is a component that models the behavior of a double layer capacitor in actual electrochemical cells, that is, an imperfect capacitor, and the impedance is represented as

$$
Z_{T}=A \text {. }
$$

Equation (8) describes the deviation from ideal capacitors. Considering $\gamma=1$ and the constant $A=1 / C$ (the inverse of capacitance), this equation describes a capacitor. For a CPE, the exponent $\gamma$ is less than one [29].

In electrochemical systems the diffusion can create an impedance called Warburg impedance [26], commonly used to describe phenomena such as diffusion, adsorption, or desorption of electroactive substances at interfaces metal/coating; this impedance depends on perturbation frequency: at high frequency a small Warburg impedance results and at low frequency a higher Warburg impedance is generated. The Warburg impedance parameter indicates the existence of diffusive processes that can be related to the release of the dissolved species. This parameter only reports the blocking ability of the passive layer, so it is not possible to know the nature of the species which are dissolved by electrochemical impedance spectroscopy technique. The equation for the infinite thickness of the Warburg impedance is given by

$$
Z_{T}=\varrho(\omega)^{-1 / 2}(1-j)
$$

where $\varrho$ is a Warburg coefficient. On a Nyquist plot the infinite Warburg impedance appears as a diagonal line with a slope of 0.5 ; on a Bode plot, the Warburg impedance exhibits a phase shift of $45^{\circ}$ [29].

Equation (9) is valid if the diffusion layer has an infinite thickness. Quite often this is not the case. If the diffusion layer is bounded, the impedance at lower frequencies no longer obeys (9). For the Warburg impedance with finite thickness, we get the form

$$
Z_{T}=\varrho(\omega)^{-1 / 2}(1-j) \tanh \left[\delta\left(\frac{j \omega}{D}\right)\right]
$$

where $\delta$ is the Nernst diffusion layer thickness; $D$ is an average value of the diffusion coefficients of the diffusing species. This equation is more general and is called finite Warburg [29].

3.1. Equivalent Representation Based on Fractal Capacitors. One of the problems of the fractional representation is the correct sizing of the physical parameters involved in the differential equation, to be consistent with dimensionality and following [12] we introduce an auxiliary parameter $\alpha$ in the following way:

$$
\frac{d}{d t} \longrightarrow \frac{1}{\alpha^{1-\gamma}}{ }_{0}^{C} D_{t}^{\gamma}, \quad n-1<\gamma \leq n
$$

or

$$
\frac{d}{d t} \longrightarrow \frac{1}{\alpha^{1-\gamma}}{ }_{0}^{\mathrm{CF}} \mathscr{D}_{t}^{\gamma}, \quad n-1<\gamma \leq n,
$$

where $n$ is an integer and when $\gamma=1$. Expressions (11) and (12) become a classical derivative; the auxiliary parameter $\alpha$ has the dimension of time (seconds). This nonlocal time is called the cosmic time in the literature [30]. Another physical and geometrical interpretation of the fractional operators is given in Moshrefi-Torbati and Hammond [31]. Parameter $\alpha$ characterizes the fractional temporal structures (components that show an intermediate behavior between a conservative system and dissipative; such components change the time constant of the system) of the fractional temporal operator [32]. In the following we will apply this idea to construct the fractional equivalent circuits and examples are analyzed.

3.2. Polarizable Electrode. The model of polarizable electrode, also known as faradaic reaction, provides a simple description of the impedance of an electrochemical reaction on electrode surfaces. The equivalent circuit is represented by Figure 1. 


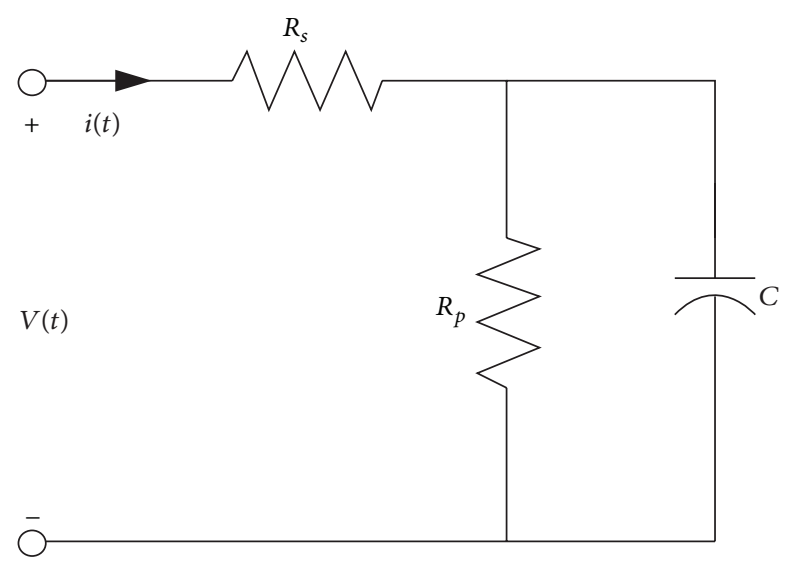

FIGURE 1: Equivalent electrical circuit for the polarizable electrode model.

Considering initial conditions equal to zero, the equivalent impedance is found by the following equation in the complex frequency domain:

$$
Z_{T}(s)=\frac{V(s)}{I(s)}
$$

Applying Kirchhoff laws to the circuit of Figure 1, we have

$$
\begin{gathered}
V=R_{s} i+V_{C}, \\
i=i_{R}+i_{C} .
\end{gathered}
$$

Before applying the Laplace transform of (15) the following considerations must be taken into account:

$$
\begin{aligned}
& i_{R}=\frac{V_{C}}{R_{p}}, \\
& i_{C}=C \frac{d V_{C}}{d t} .
\end{aligned}
$$

Consider (11); in the Caputo sense (1), (17) becomes

$$
\begin{aligned}
i_{R} & =\frac{V_{C}}{R_{p}}, \\
i_{C}(t) & =C+\frac{C}{\alpha^{1-\gamma}}{ }_{0}^{C} D_{t}^{\gamma} V_{C}(t) .
\end{aligned}
$$

Substituting (19) into (15), we obtain

$$
\begin{aligned}
V(t) & =R_{s} i(t)+V_{C}(t), \\
i(t) & =\frac{V_{C}}{R_{p}}+\frac{C}{\alpha^{1-\gamma}}{ }_{0}^{C} D_{t}^{\gamma} V_{C}(t) .
\end{aligned}
$$

Applying the Laplace transform (2) to (21), we obtain

$$
\begin{aligned}
V(s) & =R_{s} I(s)+V_{C}(s), \\
I(s) & =\frac{V_{C}(s)}{R_{p}}+\frac{C}{\alpha^{1-\gamma}} s^{\gamma} V_{C}(s) .
\end{aligned}
$$

Finally from (23) we have the fractional impedance of the circuit

$$
Z_{T}(s)=R_{s}+\frac{R_{p}}{1+\left(R_{p} C / \alpha^{1-\gamma}\right) s^{\gamma}},
$$

where $s=j \omega$.

Consider (12), in the Caputo-Fabrizio sense (3), (17) becomes

$$
\begin{aligned}
i_{R} & =\frac{V_{C}}{R_{p}}, \\
i_{C}(t) & =C+\frac{C}{\alpha^{1-\gamma}}{ }_{0}^{\mathrm{CF}} \mathscr{D}_{t}^{\gamma} V_{C}(t) .
\end{aligned}
$$

Substituting (26) into (15), we obtain

$$
\begin{aligned}
V(t) & =R_{s} i(t)+V_{C}(t), \\
i(t) & =\frac{V_{C}}{R_{p}}+\frac{C}{\alpha^{1-\gamma}}{ }_{0}^{C F} \mathscr{D}_{t}^{\gamma} V_{C}(t) .
\end{aligned}
$$

Applying the Laplace transform (5) to (28) we obtain

$$
\begin{aligned}
& V(s)=R_{s} I(s)+V_{C}(s), \\
& I(s)=\frac{V_{C}(s)}{R_{p}}+\frac{C}{\alpha^{1-\gamma}}\left[\frac{s}{s+\gamma(1-s)}\right] V_{C}(s) .
\end{aligned}
$$

Finally from (30) we have the fractional impedance of the circuit

$$
Z_{T}(s)=R_{s}+\frac{R_{p}}{1+\left(R_{p} C / \alpha^{1-\gamma}\right)(1 /(1-\gamma+\gamma / s))},
$$

where $s=j \omega$ and $\alpha^{1-\gamma}$ represent the fractional components of the system. Equations (24) and (31) are the result of applying the fractional temporal operator of Caputo and Caputo-Fabrizio type in (17) for the current in the capacitor; this general representation includes an arbitrary constant, $\alpha$, which can be considered its own electrochemical parameter. In the particular case of $\alpha=R_{p} C$, (24) is reduced to the Cole model (7). Equations (24) and (31) presented here preserve the dimensionality of the studied system for any value of the exponent of the fractional derivative. On the other hand, if $\gamma=1$ in (24) and (31), we obtain an ideal RC circuit and the Markovian nature of the model is recovered.

Consider that the values of the parameters of the circuit shown in Figure 1 correspond to $R_{s}=100 \Omega, C=1^{-4} \mathrm{~F}$, and $R_{p}=200 \Omega$ and $R_{p}=300 \Omega$. Figures $2(\mathrm{a}), 2(\mathrm{~b}), 2(\mathrm{c})$, and $2(\mathrm{~d})$ show the Nyquist and Bode plots for (24).

Now consider that the values of the parameters of the circuit shown in Figure 1 correspond to $R_{s}=100 \Omega, C=$ $1^{-4} \mathrm{~F}$, and $R_{p}=200 \Omega$ and $R_{p}=300 \Omega$. Figures $3(\mathrm{a}), 3(\mathrm{~b})$, $3(\mathrm{c})$, and 3(d) show the Nyquist and Bode plots for (31).

Several studies use equivalent circuits considering pure capacitances for adjusting the impedance spectra and thus describe phenomena as deterioration of materials due to its porosity or the effects of exposure and surface preparation 


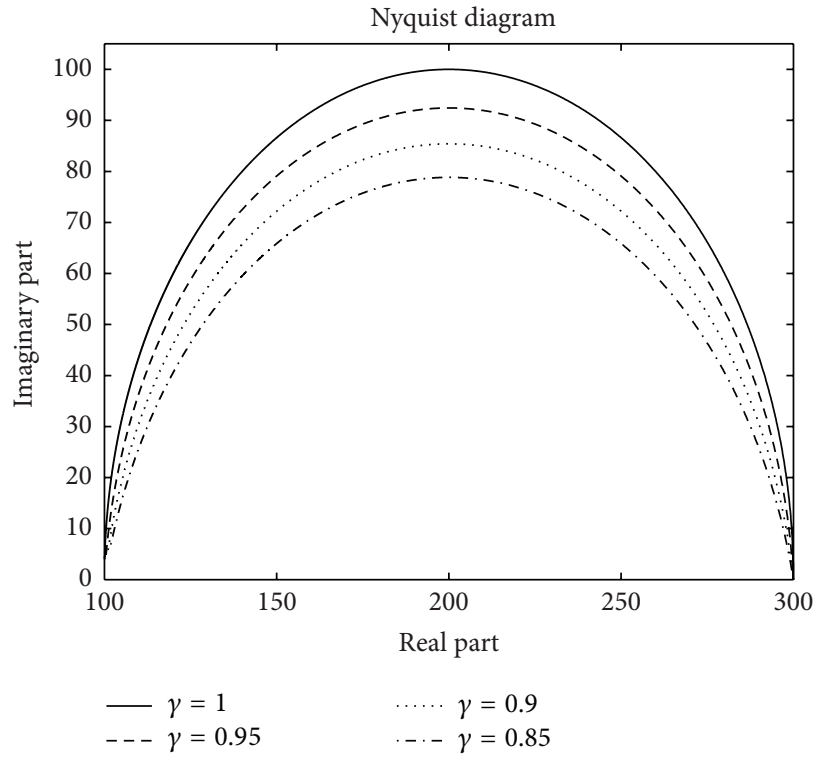

(a)

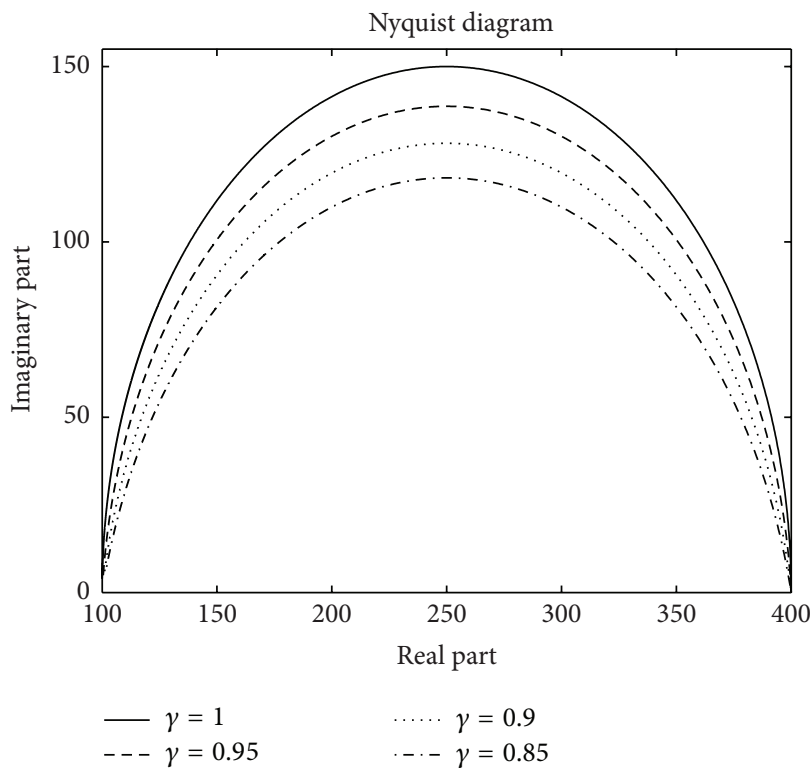

(c)
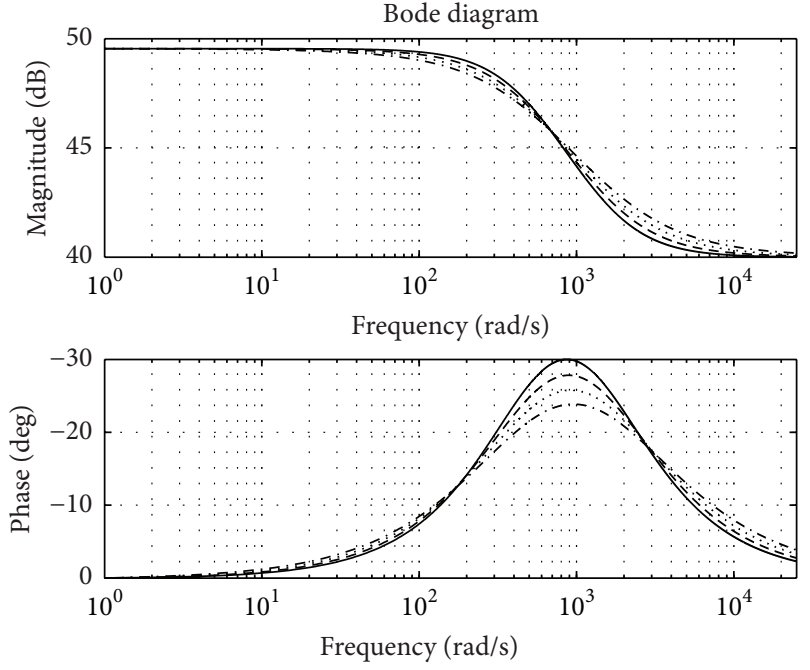

$\begin{aligned} \gamma & =1 & \cdots & \gamma=0.9 \\ --\gamma & =0.95 & \cdots-\gamma & =0.85\end{aligned}$

(b)
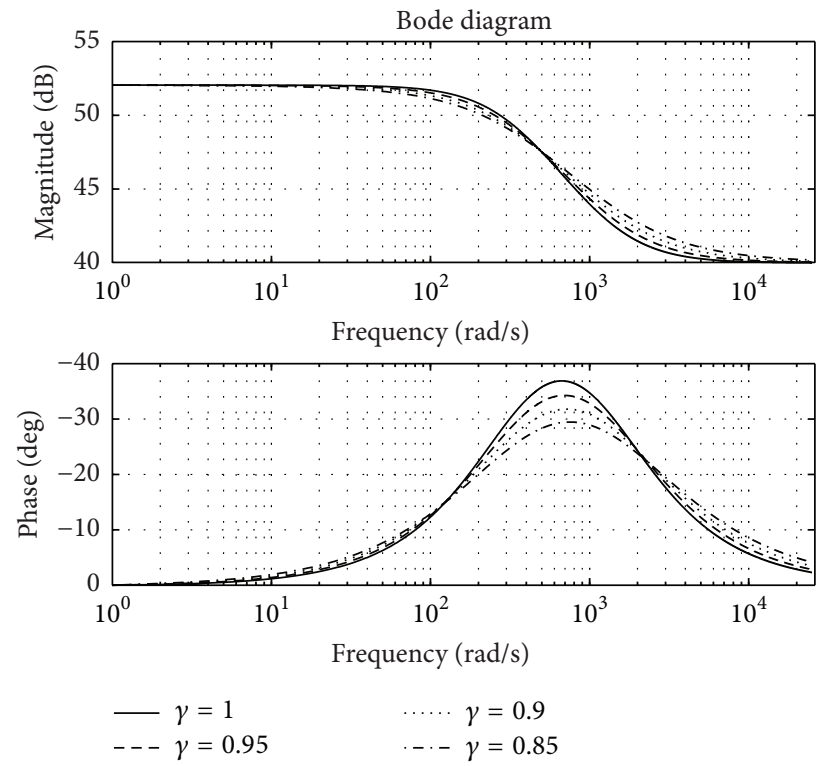

(d)

FIGURE 2: Nyquist and Bode diagram for the model of polarizable electrode, Caputo derivative approach, in (a) and (b): $R_{s}=100 \Omega, C=1^{-4} \mathrm{~F}$, and $R_{p}=200 \Omega$ for $\gamma=1, \gamma=0.95, \gamma=0.9$, and $\gamma=0.85$; for (c) and (d), $R_{s}=100 \Omega, C=1^{-4} \mathrm{~F}$, and $R_{p}=300 \Omega$ for $\gamma=1, \gamma=0.95, \gamma=0.9$, and $\gamma=0.85$.

of substrates on the impedances [33-36]. The electrical capacitance has information on the conductive properties of materials and their chemical composition. Recently, several authors have replaced this pure capacitance by a CPE, because this has been considered an improvement in the settings of the theoretical equations regarding experimental results [37-39], for example, in predicting the useful life of coatings and surface roughness heterogeneity resulting from the presence of impurities, fractality, dislocations, adsorption of inhibitors, or formation of porous layers [40-47]. Replacing the capacitor $C$ by a CPE adds a pseudocapacitive constant to the circuit and causes the attenuation of the imaginary part of the impedance causing a flattening of the semicircles in the Nyquist diagrams (this is their main feature); see Figures 2(a) and 2(c) for the Caputo approach and Figures 3(a) and 3(c) for the Caputo-Fabrizio approach. These figures allow seeing the effect of varying the values of the pseudocapacitive constants: in the range $\gamma \in(0.85 ; 1)$ we have the behavior of a CPE and when $\gamma=1$ the values correspond to pure capacitances. In this range, the order of the derivative transforms the capacitor $C_{p}$ into a CPE. When increasing the value of the pseudocapacitive constants, this also increases the imaginary part of the impedance and this changes the time constants of the system [32], which 


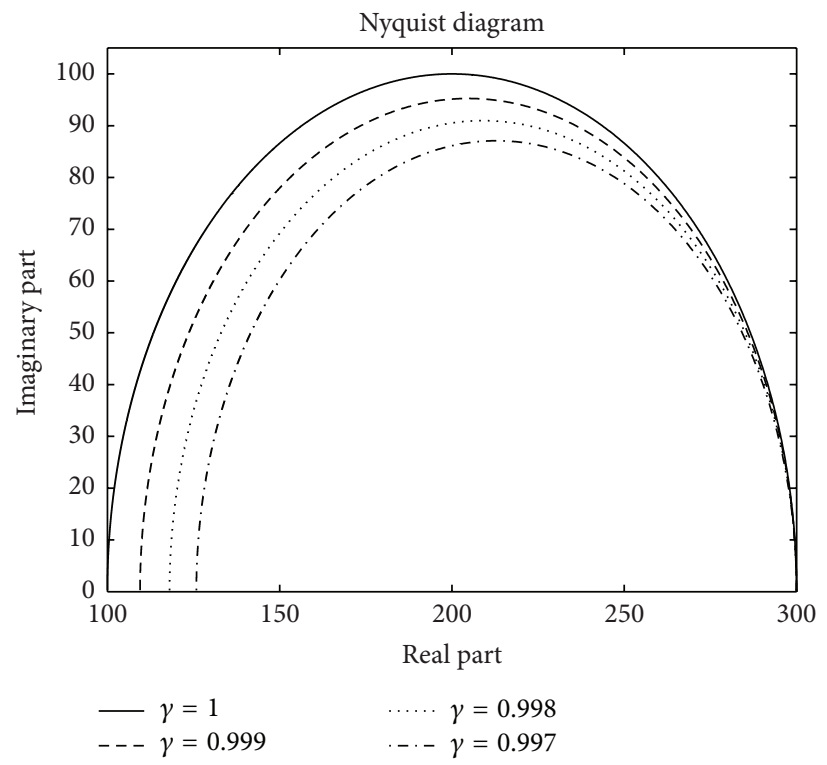

(a)

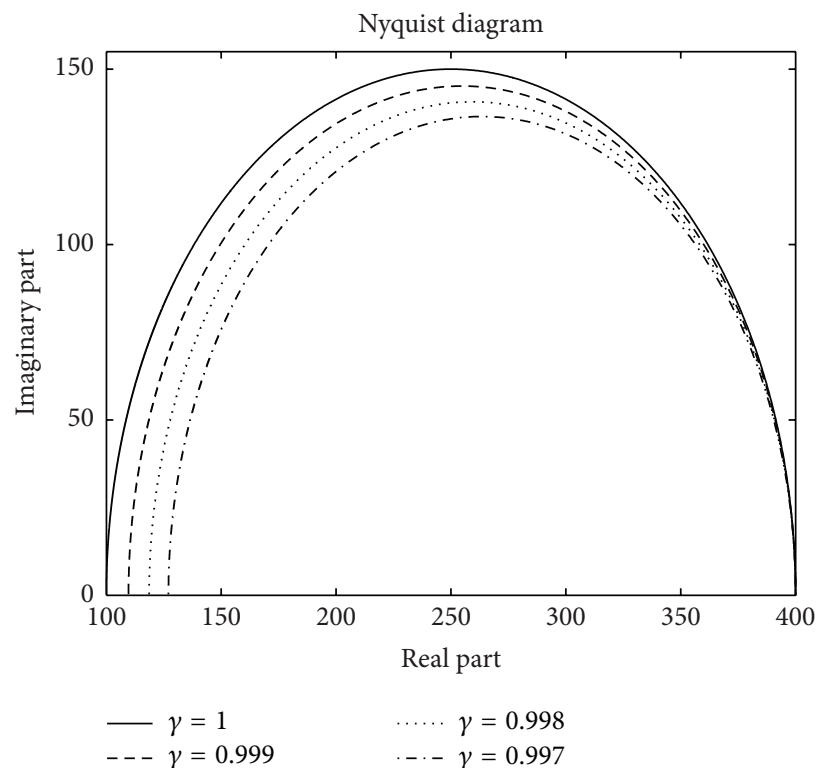

(c)
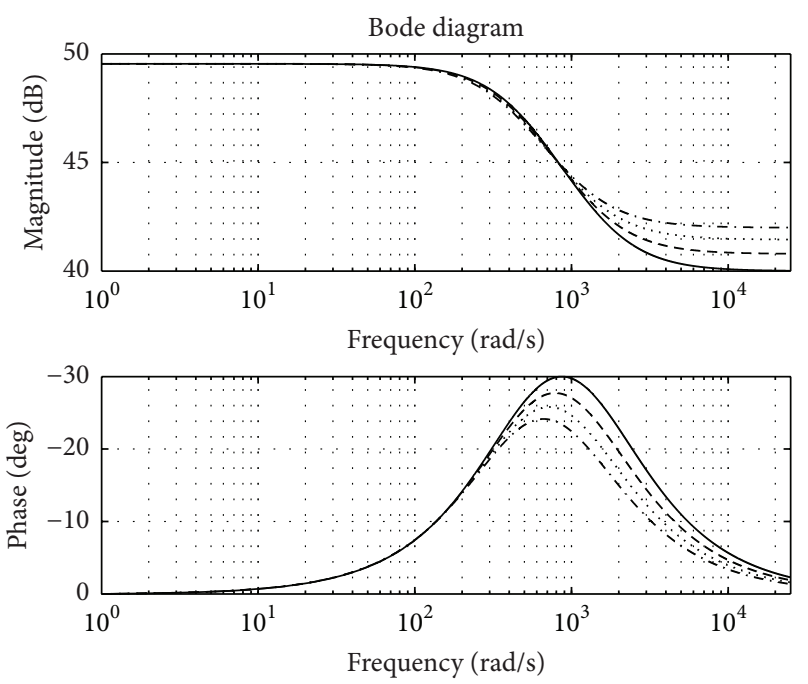

$-\gamma=1$

$--\gamma=0.999$

$\cdots, \gamma=0.998$

-.. $\gamma=0.997$

(b)
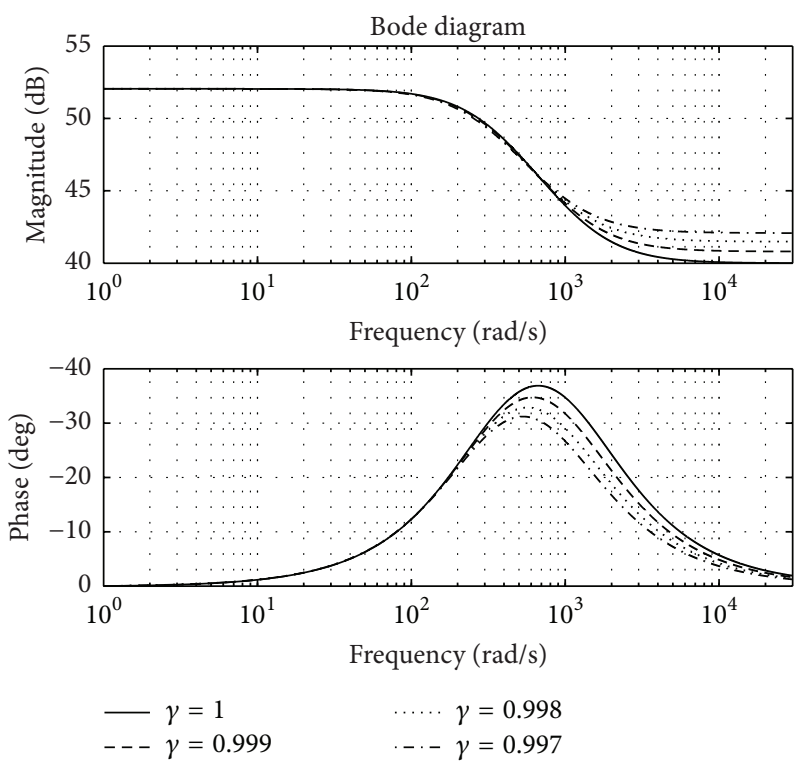

(d)

Figure 3: Nyquist and Bode diagram for the model of polarizable electrode, Caputo-Fabrizio derivative approach, in (a) and (b): $R_{s}=100 \Omega$, $C=1^{-4} \mathrm{~F}$, and $R_{p}=200 \Omega$ for $\gamma=1, \gamma=0.999, \gamma=0.998$, and $\gamma=0.997$; for (c) and (d), $R_{s}=100 \Omega, C=1^{-4} \mathrm{~F}$, and $R_{p}=300 \Omega$, for $\gamma=1$, $\gamma=0.999, \gamma=0.998$, and $\gamma=0.997$.

correspond to creating fractal structures for particular values of $\gamma$. In the Caputo-Fabrizio approach it is noted that the value of the resistance series is modified, showing the existence of heterogeneities in this component. In Figures 2(b) and 2(d) for the Caputo approach and Figures 3(b) and 3(d) for the Caputo-Fabrizio approach, the Bode plots exhibit changes in the cutoff frequency, hence the phase shift and the decrease of the magnitude. For the range of frequency 10 to $10000 \mathrm{rad} / \mathrm{s} \mathrm{a}$ shift in the magnitude and phase is shown, which implies that the proposed circuit better defines these frequencies. We see that, by increasing the frequency from the cutoff frequency, the magnitude decreases, while for frequencies below the cutoff magnitude it is almost constant. This means that the current through the circuit is very high with decreasing frequency and is very low when the frequency increases. The current flowing through these resistors at low frequencies presents dissipative effects that correspond to the nonlinear situation of the physical process (realistic behavior that is nonlocal in time), for example, the ohmic friction, which raises the temperature and therefore the kinetic energy of the molecules of the system [48]. Concerning the phase, we have that by increasing frequency the displacement current and polarization are very much increased. In this context, the need for CPE has been attributed by some authors to 


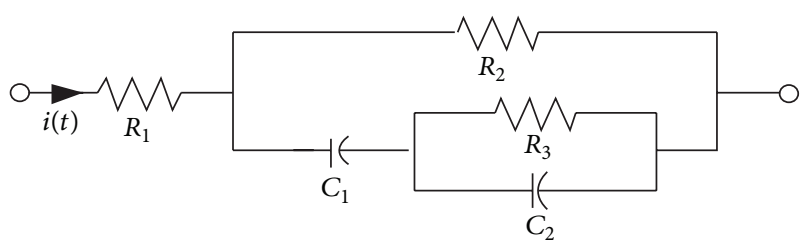

FIGURE 4: Equivalent electrical circuit for the heterogeneous reaction model.

the presence of roughness, corrosion products, changes in the morphology of the material, or heterogeneous surfaces.

3.3. Heterogeneous Reaction. This model describes a heterogeneous reaction which occurs in two stages with absorption of intermediates products and absence of diffusion limitations. This circuit has been commonly used to model a porous electrode coating or a defective electrolyte interface and has recently been applied in the assessment of passive metalelectrolyte or metal-electrolyte interfaces with hard coating $[49,50]$. The equivalent circuit is represented by Figure 4 .
This model has also been used in the study of coated metals. In this case, $C_{1}$ represents the capacitance of the coating; this value is smaller than a capacitance represented by a CPE; $R_{2}$ represents the pore resistance; it is the resistance of ion conducting paths developed in the coating. These paths may be physical pores filled with electrolyte; this electrolyte solution can be very different than the bulk solution outside of the coating; $C_{2}$ (double layer capacitance) and $R_{3}$ (charge transfer reaction) represent the interface between this pocket of solution and the bare metal.

Following the same methodology from previous example, the fractional impedance in the Caputo sense of this circuit is

$$
\begin{aligned}
& Z_{T}(s)=R_{1} \\
& \quad+\frac{R_{3}}{\left(R_{3} C_{1} / \alpha_{\gamma}^{1-\gamma}\right) s^{\gamma}+1 /\left(R_{2} / R_{3}+1 /\left(\left(R_{3} C_{2} / \alpha_{\beta}^{1-\beta}\right) s^{\beta}+1\right)\right)} .
\end{aligned}
$$

In the Caputo-Fabrizio sense the fractional impedance is given by

$$
Z_{T}(s)=R_{1}+\frac{R_{3}}{\left(R_{3} C_{1} / \alpha_{\gamma}^{1-\gamma}\right)(1 /(1-\gamma+\gamma / s))+1 /\left(R_{2} / R_{3}+1 /\left(\left(R_{3} C_{2} / \alpha_{\beta}^{1-\beta}\right)(1 /(1-\beta+\beta / s))+1\right)\right)}
$$

where $s=j \omega$, in (32) and (33), $\alpha_{\gamma}^{1-\gamma}$ and $\alpha_{\beta}^{1-\beta}$ represents the fractional components of the system. This general representation includes an arbitrary constant, $\alpha$, which can be considered its own electrochemical parameter. In the case of $\alpha_{\gamma}$ the physical parameters involved are $R_{3} C_{1}$ and for $\alpha_{\beta}$ they are $R_{3} C_{2}$, the time constant of the system. Equations (32) and (33) presented here preserve the dimensionality of the studied system for any value of the exponent of the fractional derivative; when $\gamma=\beta=1$, we recover the Markovian nature of the model.

Consider that the values of the parameters of the circuit shown in Figure 4 correspond to $R_{1}=50 \Omega, R_{2}=100 \Omega$, $R_{3}=200 \Omega, C_{1}=1^{-3} \mathrm{~F}, C_{2}=1^{-2} \mathrm{~F}$, and $C_{2}=3^{-3} \mathrm{~F}$. Figures 5(a), 5(b), 5(c), and 5(d) show the Nyquist and Bode plots for (32).

Now consider that the values of the parameters of the circuit shown in Figure 4 correspond to $R_{1}=50 \Omega, R_{2}=$ $100 \Omega, R_{3}=200 \Omega, C_{1}=1^{-3} \mathrm{~F}, C_{2}=1^{-2} \mathrm{~F}$, and $C_{2}=3^{-3} \mathrm{~F}$. Figures 6(a), 6(b), 6(c), and 6(d) show the Nyquist and Bode plots for (33).

Some authors make changes to the ideal capacitors including elements of Warburg $[49,50]$; these elements are represented in our circuit maintaining the order of the derivatives fixed at a value of $\gamma=\beta=1 / 2$. For Caputo and CaputoFabrizio approach, Figures 7(a) and 7(c), respectively, show the resulting Nyquist diagrams considering the order of the derivative for both capacitors at $1 / 2$. From these figures it can be seen that for high frequency the impedance is small because the reagents must move away from the surface.
The low-frequency disturbances allow the movement of distant molecules.

The circuit shown in Figure 4 has also been used with success in the description of titanium alloys, considering carbon coatings, silicon oxide, and titanium oxide; in such applications it is generally considered that the coating presents a porous outer sublayer and a denser internal sublayer $[49,51]$. In Figure 7(c) it is observed that the resistance value decreases, which can provide valuable information on the characteristics of a coating film.

3.4. Limited Diffusion, Warburg Element. This model describes the polarization of an electrode considering limiting the diffusion. This circuit models a cell where polarization is due to a combination of kinetic and diffusion processes, $R_{s}$ is the resistance of the electrolyte solution, CPE is the imperfect capacitor, $R_{p}$ is the electron-transfer resistance, and $W$ is the Warburg element due to diffusion of the redox couple to the interface from the bulk of the electrolyte [52]. The equivalent circuit is represented by Figure 8 .

Following the same methodology from previous example, the fractional impedance in the Caputo sense of this circuit is

$$
\begin{aligned}
& Z_{T}(s) \\
& =R_{s} \\
& \quad+\frac{R_{p}}{\left(R_{p} C_{p} / \alpha_{\gamma}^{1-\gamma}\right) s^{\gamma}+1 /\left(1+1 /\left(R_{p} C_{s} / \alpha_{\beta}^{1-\beta}\right) s^{\beta}\right)} .
\end{aligned}
$$




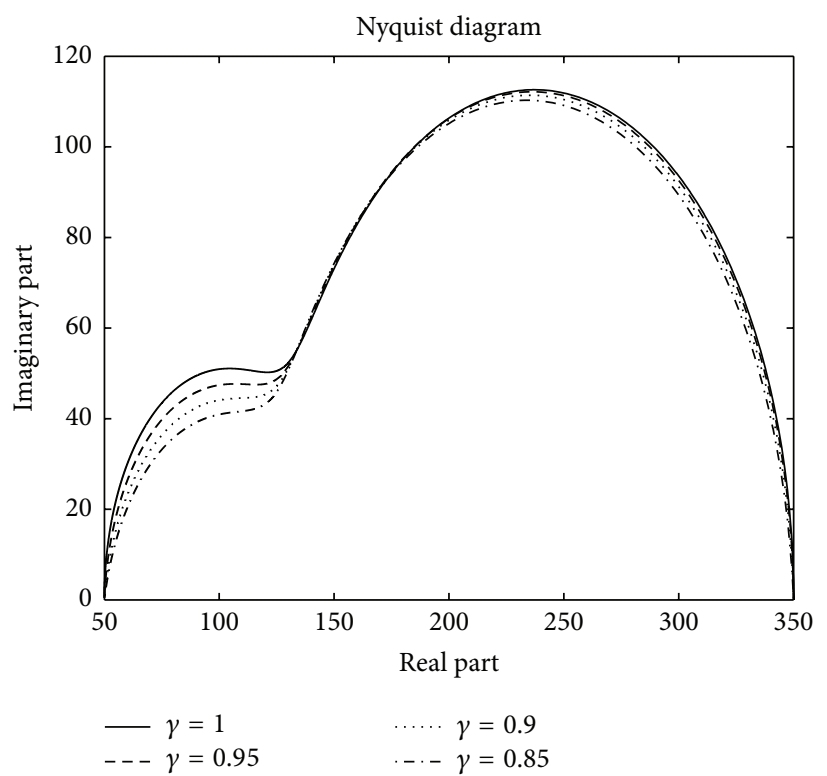

(a)

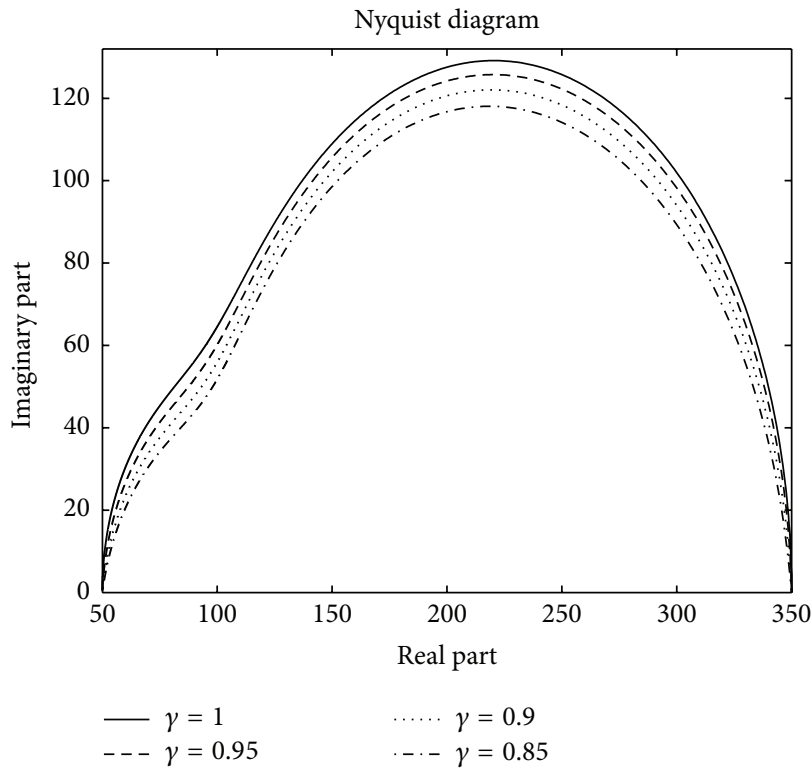

(c)
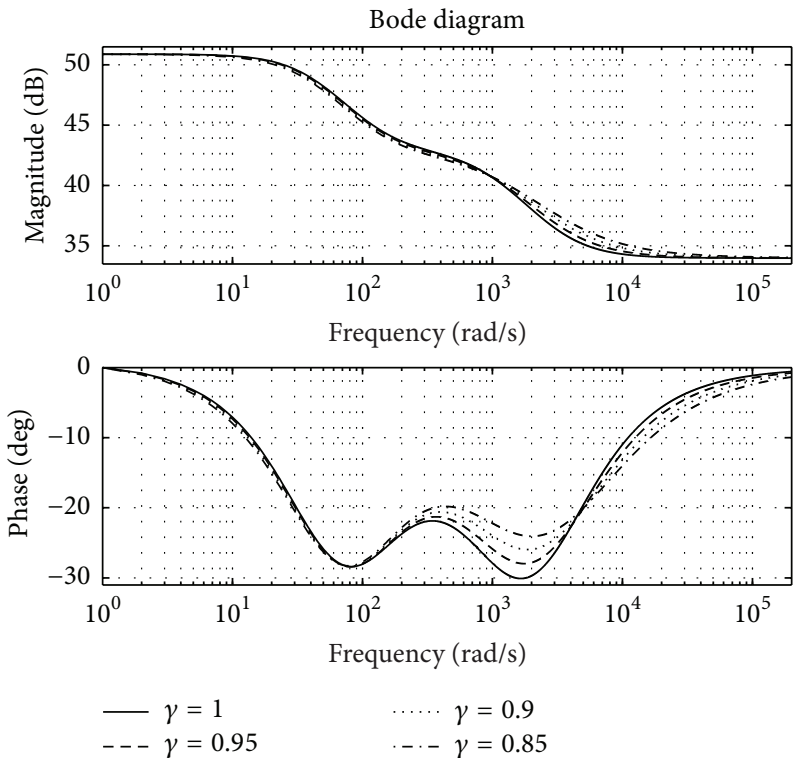

(b)
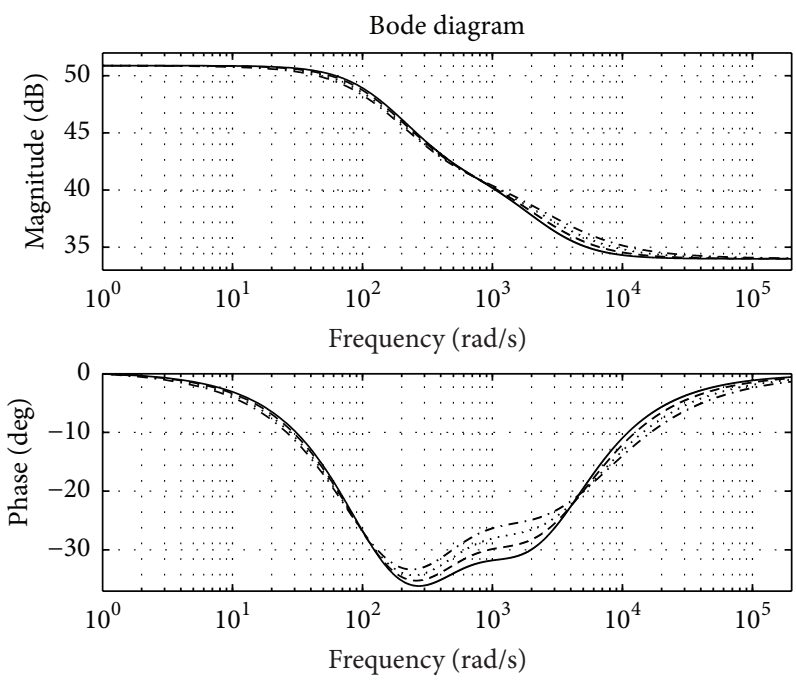

$\begin{aligned} \gamma & =1 \\ -\gamma & \cdots\end{aligned}$

(d)

FIGURE 5: Nyquist and Bode diagram for the heterogeneous reaction model, Caputo derivative approach, in (a) and (b): $R_{1}=50 \Omega, R_{2}=100 \Omega$, $R_{3}=200 \Omega, C_{1}=1^{-3} \mathrm{~F}$, and $C_{2}=1^{-2} \mathrm{~F}$ for $\gamma=1, \gamma=0.95, \gamma=0.9$, and $\gamma=0.85$; for (c) and (d), $R_{1}=50 \Omega, R_{2}=100 \Omega, R_{3}=200 \Omega$, $C_{1}=1^{-3} \mathrm{~F}$, and $C_{2}=3^{-3} \mathrm{~F}$ for $\gamma=1, \gamma=0.95, \gamma=0.9$, and $\gamma=0.85$. The capacitor $C_{2}$ is considered ideal, $\beta=1$.

In the Caputo-Fabrizio sense the fractional impedance is given by

$$
Z_{T}(s)=R_{s}+\frac{R_{p}}{\left(R_{p} C_{p} / \alpha_{\gamma}^{1-\gamma}\right)(1 /(1-\gamma+\gamma / s))+1 /\left(1+1 /\left(R_{p} C_{s} / \alpha_{\beta}^{1-\beta}\right)(1 /(1-\beta+\beta / s))\right)},
$$

where $s=j \omega$, in (34) and (35), and $\alpha_{\gamma}^{1-\gamma}$ and $\alpha_{\beta}^{1-\beta}$ represent the fractional components of the system. This general representation includes an arbitrary constant, $\alpha$, which can be considered its own electrochemical parameter. In the case of $\alpha_{\gamma}$ the physical parameters involved are $R_{p} C_{p}$ for $\alpha_{\gamma}$ and for $\alpha_{\beta}$ are $R_{p} C_{s}$, the time constant of the system. For (34) and 


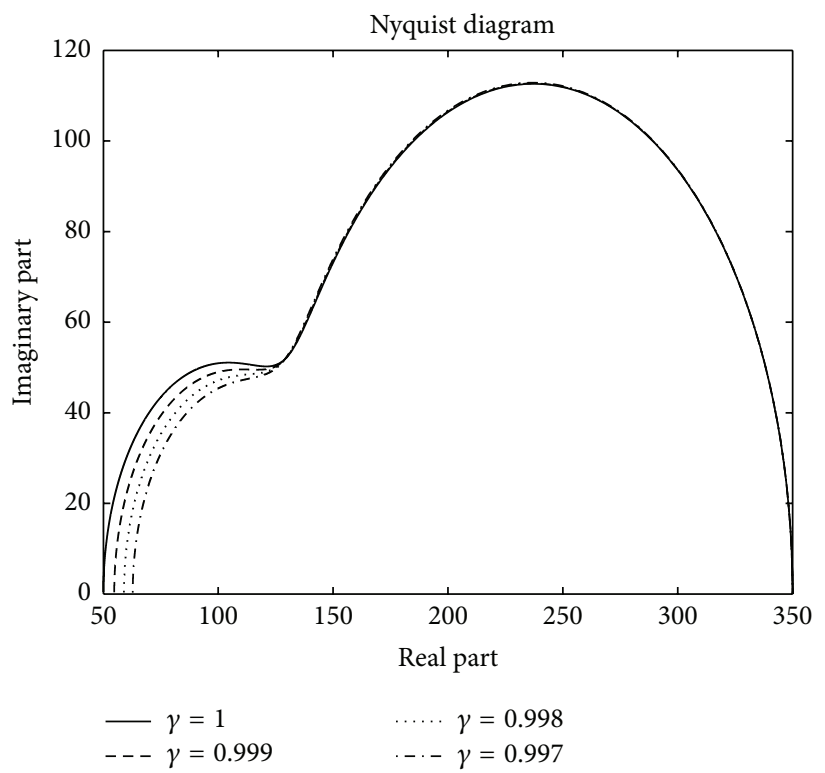

(a)

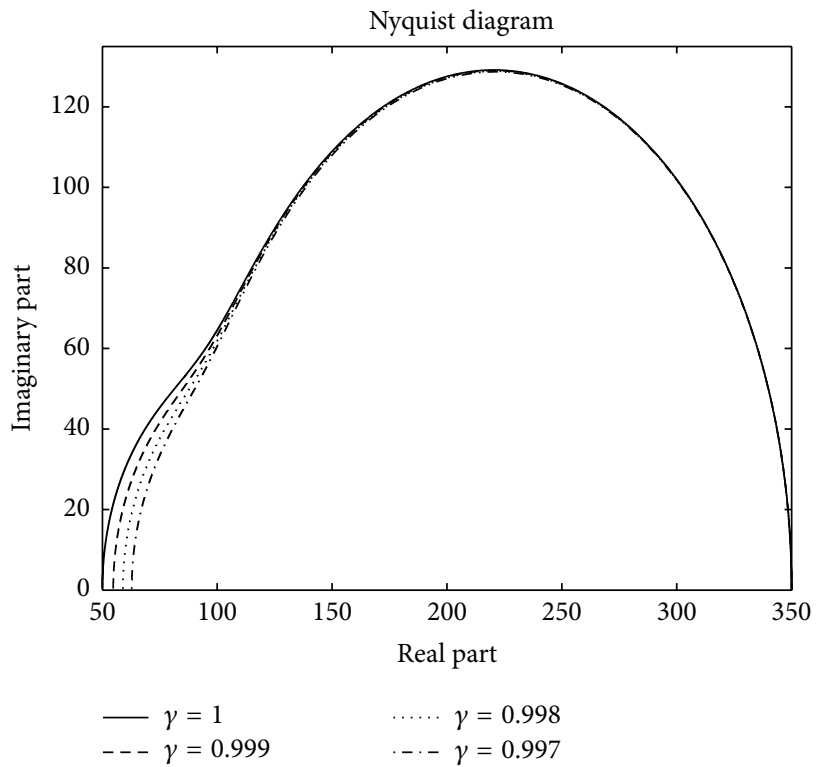

(c)
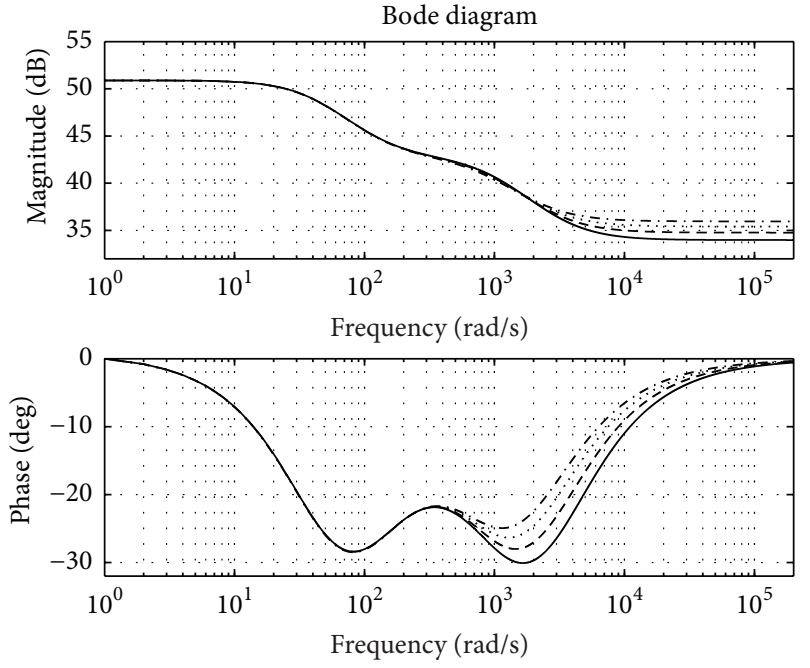

$$
\begin{aligned}
\gamma & =1 & \cdots \cdot \gamma & =0.998 \\
--\gamma & =0.999 & --\gamma & =0.997
\end{aligned}
$$

(b)
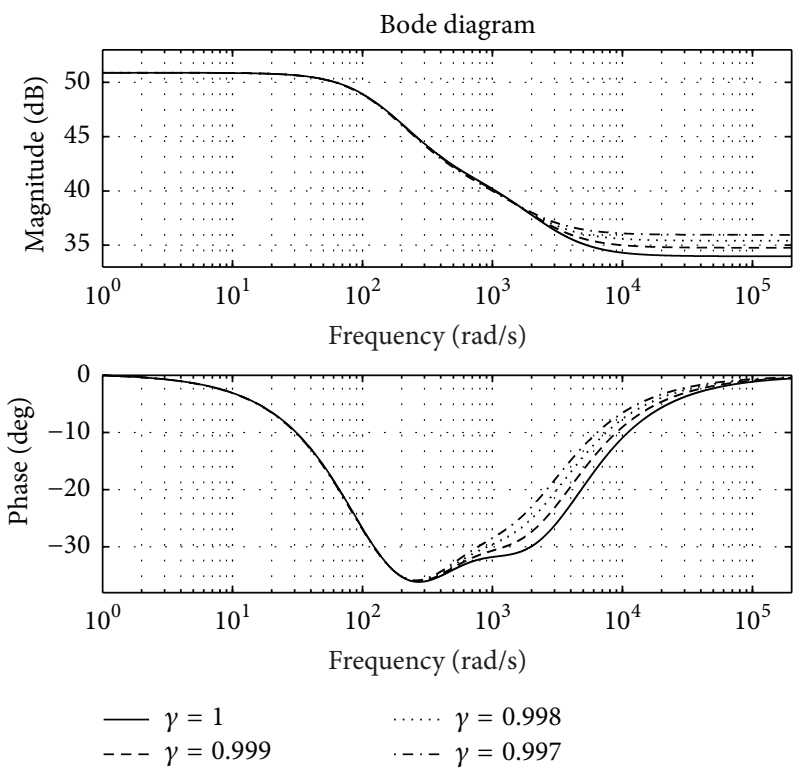

(d)

Figure 6: Nyquist and Bode diagram for the heterogeneous reaction model, Caputo-Fabrizio derivative approach, in (a) and (b): $R_{1}=50 \Omega$, $R_{2}=100 \Omega, R_{3}=200 \Omega, C_{1}=1^{-3} \mathrm{~F}$, and $C_{2}=1^{-2} \mathrm{~F}$ for $\gamma=1, \gamma=0.999, \gamma=0.998$, and $\gamma=0.997$; for (c) and $(\mathrm{d}), R_{1}=50 \Omega, R_{2}=100 \Omega$, $R_{3}=200 \Omega, C_{1}=1^{-3} \mathrm{~F}$, and $C_{2}=3^{-3} \mathrm{~F}$ for $\gamma=1, \gamma=0.999, \gamma=0.998$, and $\gamma=0.997$. The capacitor $C_{2}$ is considered ideal, $\beta=1$.

(35) when $\gamma=\beta=1$, we recover the Markovian nature of the model.

The capacitor $C$ and Warburg element $W$ shown in Figure 8 are replaced by two fractional capacitors $C_{p}$ and $C_{s}$, respectively. The capacitor $C_{s}$ is assigned an exponent $\beta=1 / 2$, causing replacement of pure capacitance and causing thus an impedance according to an infinite diffusion layer or Warburg impedance. This element arises from onedimensional diffusion of an ionic species to the electrode [18]. The capacitance of fractional order $C_{p}$ can be modeled in the range $0<\gamma \leq 1$, causing that this pure capacitance represents a CPE or imperfect capacitance. Equations (34) and (35) presented here preserve the dimensionality of the studied system for any value of the exponent of the fractional derivative. The equivalent circuit shown in Figure 8 has been used to model phenomena of corrosion stability in the physiological environment such as the simulated body fluid; $R_{s}$ represents the electrolyte resistance, $R_{p}$ represents the coating pore resistance, and the capacitances represent the frequencydependent electrochemical phenomena, such as the coating 


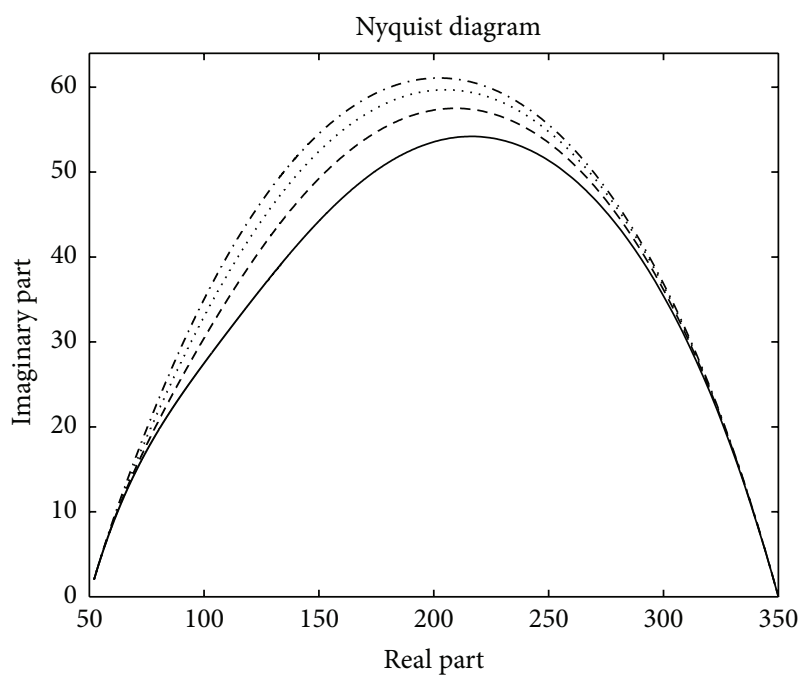

(a)

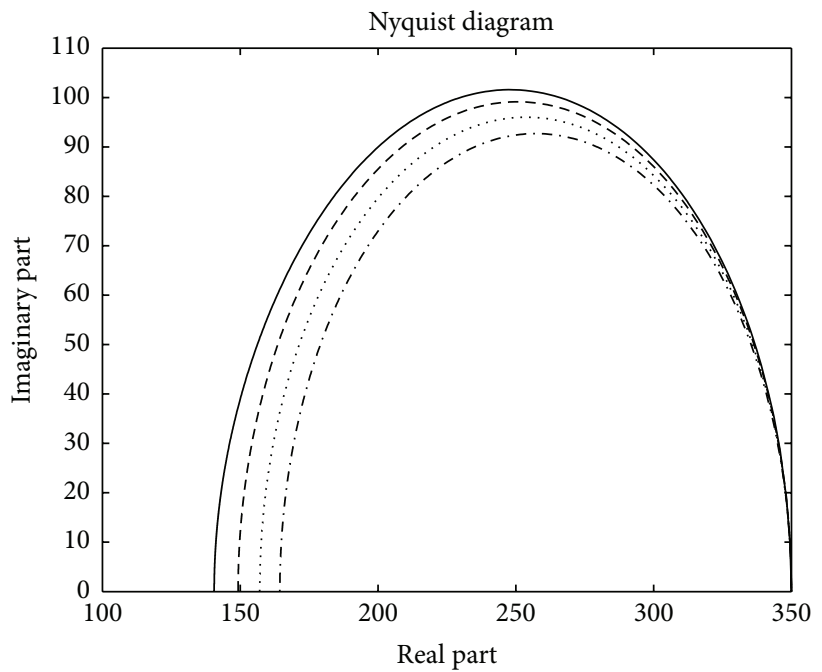

(c)
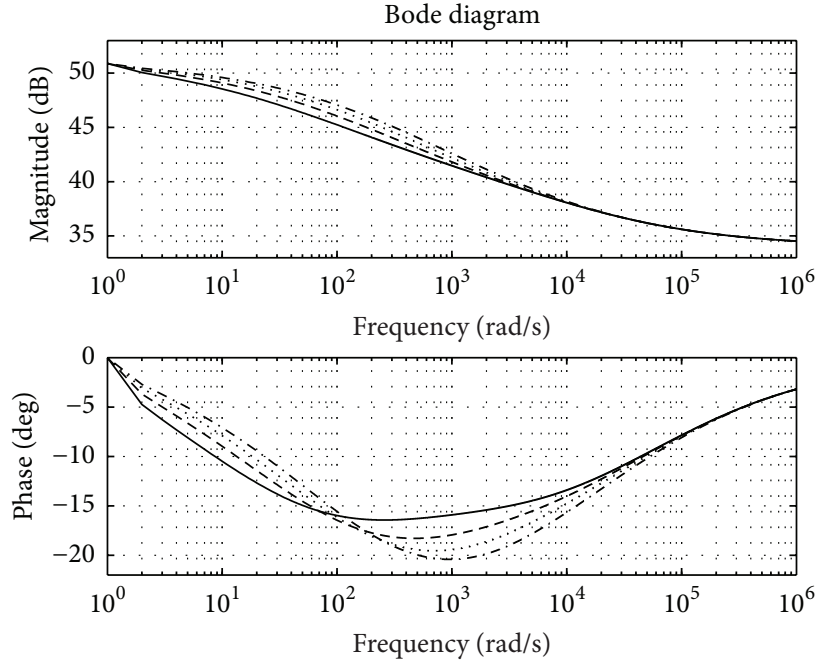

(b)
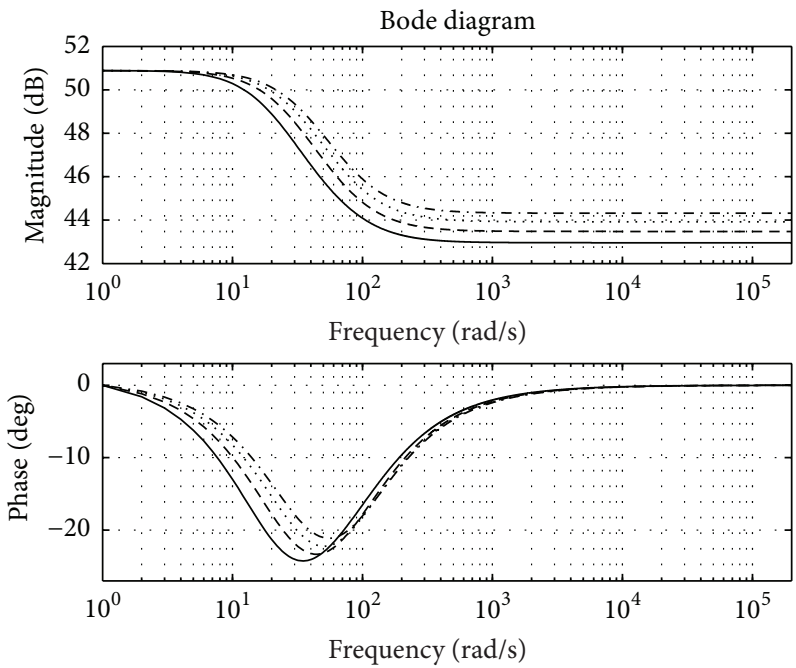

(d)

FIgURE 7: Nyquist and Bode diagram for the heterogeneous reaction model, Caputo approach in (a) and (b) and Caputo-Fabrizio approach in (c) and (d). For (a) and (b), $R_{1}=50 \Omega, \mathrm{R}_{2}=100 \Omega, R_{3}=200 \Omega, C_{1}=1^{-3} \mathrm{~F}, C_{2}=1^{-2} \mathrm{~F}$ (solid line), $C_{2}=3^{-3} \mathrm{~F}$ (dash line), $C_{2}=3^{-3} \mathrm{~F}$ (dot line), and $C_{2}=3^{-4} \mathrm{~F}$ (dash-dot line). For the capacitor $C_{1}, \gamma=1 / 2$; for the capacitor $C_{2}, \beta=1 / 2$. For (c) and (d), $R_{1}=50 \Omega, R_{2}=100 \Omega$, $R_{3}=200 \Omega, C_{1}=1^{-3} \mathrm{~F}, C_{2}=1^{-2} \mathrm{~F}$ (solid line), $C_{2}=3^{-3} \mathrm{~F}$ (dash line), $C_{2}=3^{-3} \mathrm{~F}$ (dot line), and $C_{2}=3^{-4} \mathrm{~F}$ (dash-dot line). For the capacitor $C_{1}, \gamma=1 / 2$; for the capacitor $C_{2}, \beta=1 / 2$.
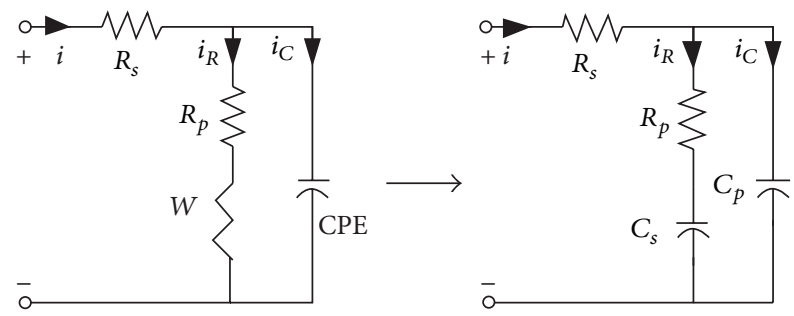

FIGURE 8: Equivalent electrical circuit for the limited diffusion model.

impedance and passive oxide film capacitance. CPE is used in these models to compensate the nonhomogeneity in the system [53].
Consider that the values of the parameters of the circuit shown in Figure 8 correspond to $R_{s}=100 \Omega, R_{p}=5000 \Omega$, $C_{s}=3^{-6} \mathrm{~F}, C_{s}=3^{-7} \mathrm{~F}$, and $C_{p}=1^{-3} \mathrm{~F}$. Figures 9(a), 9(b), 9(c), and 9(d) show the Nyquist and Bode plots for (34).

Now consider that the values of the parameters of the circuit shown in Figure 8 correspond to $R_{s}=100 \Omega, R_{p}=$ $5000 \Omega, C_{s}=3^{-6} \mathrm{~F}, C_{s}=3^{-7} \mathrm{~F}$, and $C_{p}=1^{-3} \mathrm{~F}$. Figures 10(a), 10(b), 10(c), and 10(d) show the Nyquist and Bode plots for (35).

Replacing capacitor $C$ by a CPE introduces a pseudocapacitive constant at circuit and causes the attenuation of the imaginary part of the impedance causing a flattening of the semicircles in the Nyquist diagrams; see Figure 9(a) for the Caputo approach and Figure 10(a) for the Caputo-Fabrizio 


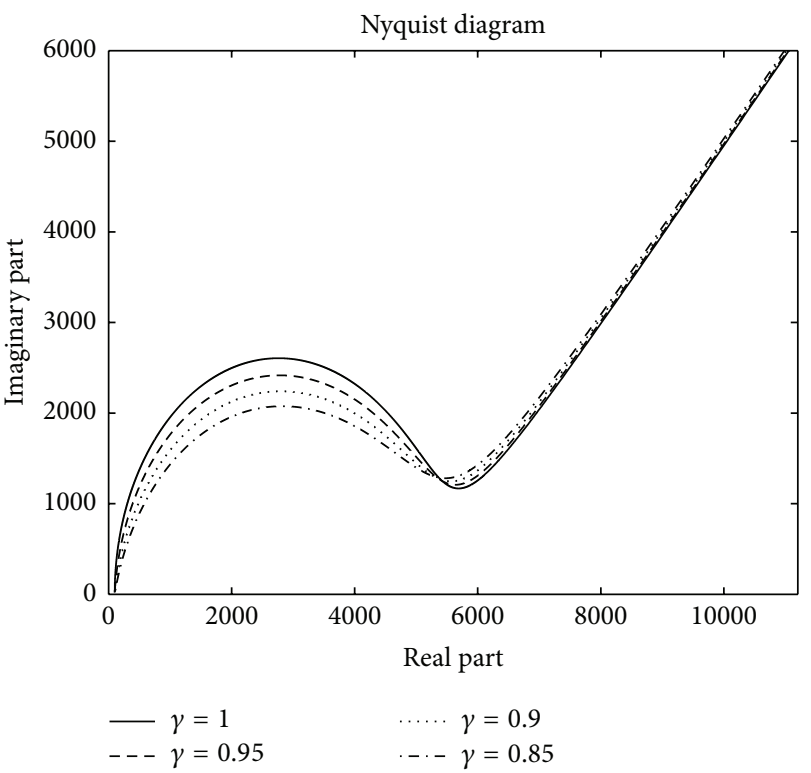

(a)

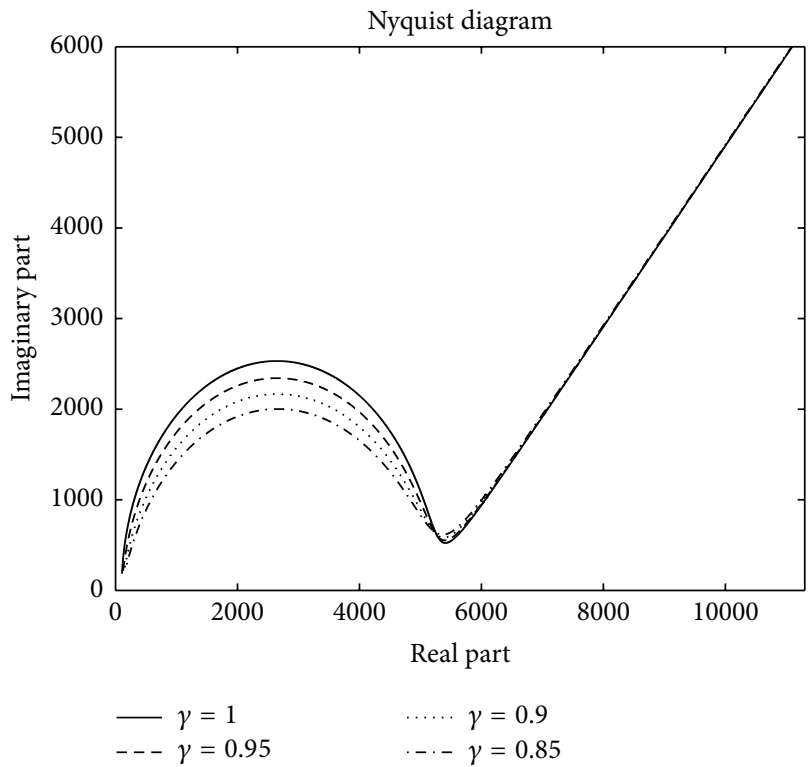

(c)
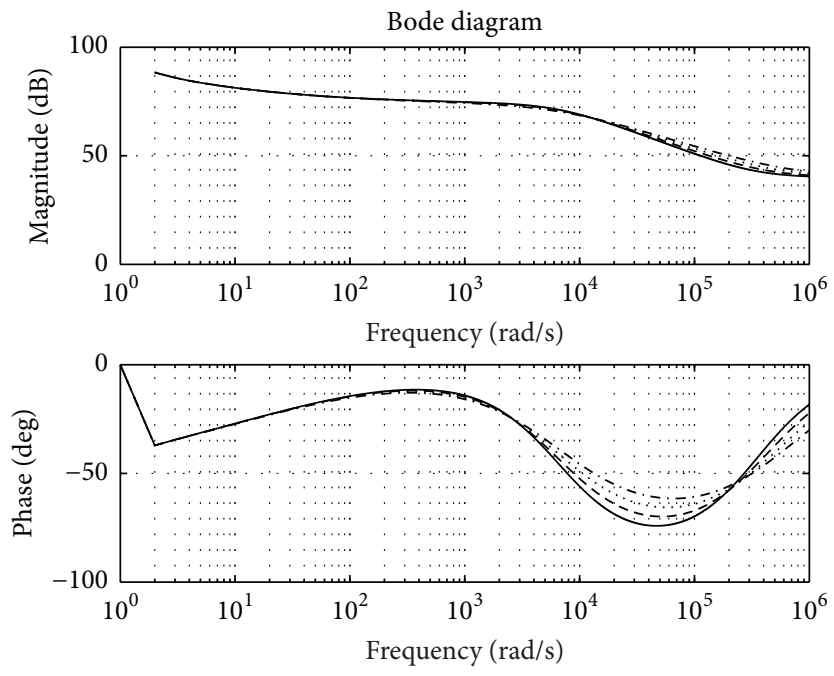

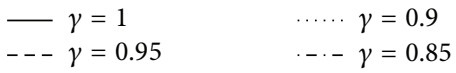

(b)
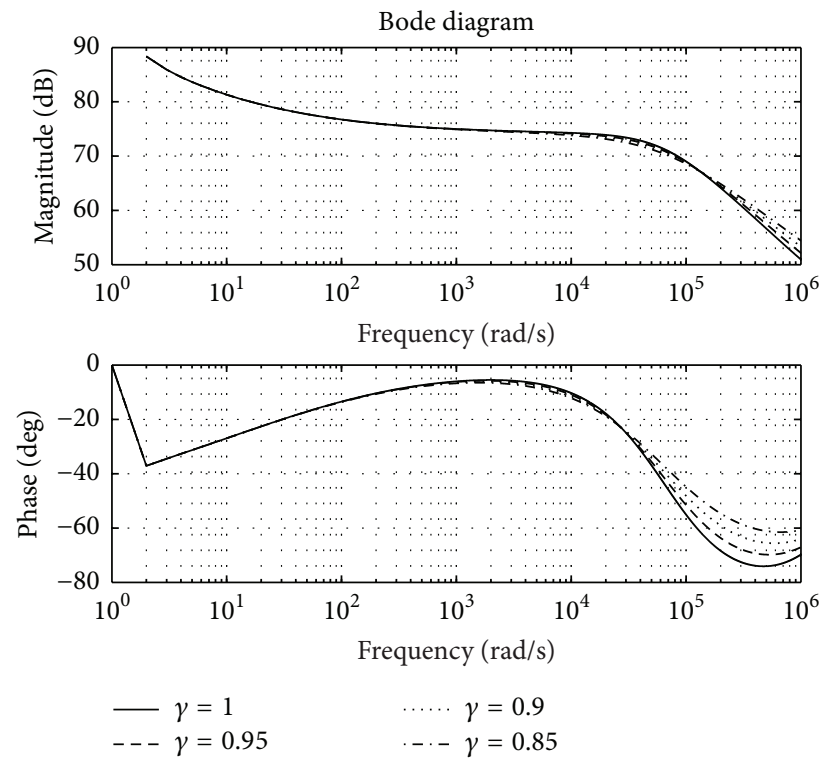

(d)

Figure 9: Nyquist and Bode diagram for the limited diffusion model, Caputo derivative approach, in (a) and (b): $R_{s}=100 \Omega, R_{p}=5000 \Omega$, $C_{s}=3^{-6} \mathrm{~F}$, and $C_{p}=1^{-3} \mathrm{~F}$ for $\beta=1 / 2$ corresponding to $C_{s}, \gamma=1, \gamma=0.95, \gamma=0.9$, and $\gamma=0.85$; for (c) and (d), $R_{p}=5000 \Omega, C_{s}=3^{-7} \mathrm{~F}$, and $C_{p}=1^{-3} \mathrm{~F}$ for $\beta=0.4$ corresponding to $C_{s}, \gamma=1, \gamma=0.95, \gamma=0.9$, and $\gamma=0.85$.

approach. Increasing the value of the pseudocapacitive constant implies an increase in the contribution of the imaginary part of the impedance and this changes the system's time constants [32], creating fractal structures which correspond to particular values of $\gamma$. In Figures 9(b), 9(d), 10(b), and 10(d) for the Caputo and Caputo-Fabrizio approach, respectively, the Bode plots show variations in the cutoff frequency, hence the phase shift and the decrease of the magnitude.

If $0<\beta<0.5$, then we have a model that geometrically describes the activation surface inhomogeneities or deviations from linear diffusion processes. In our example $\beta=0.4$.
This occurs naturally when the diffusion occurs in a dilute solution or in the case that the spread does not obey the laws of Fick; see Figures 9(c) and 10(c).

\section{Conclusions}

An impedance spectrum which is obtained in response to the small amplitude signal excitation is often interpreted in terms of an equivalent electrical circuit. This one is based on a physical model that may represent and characterize 


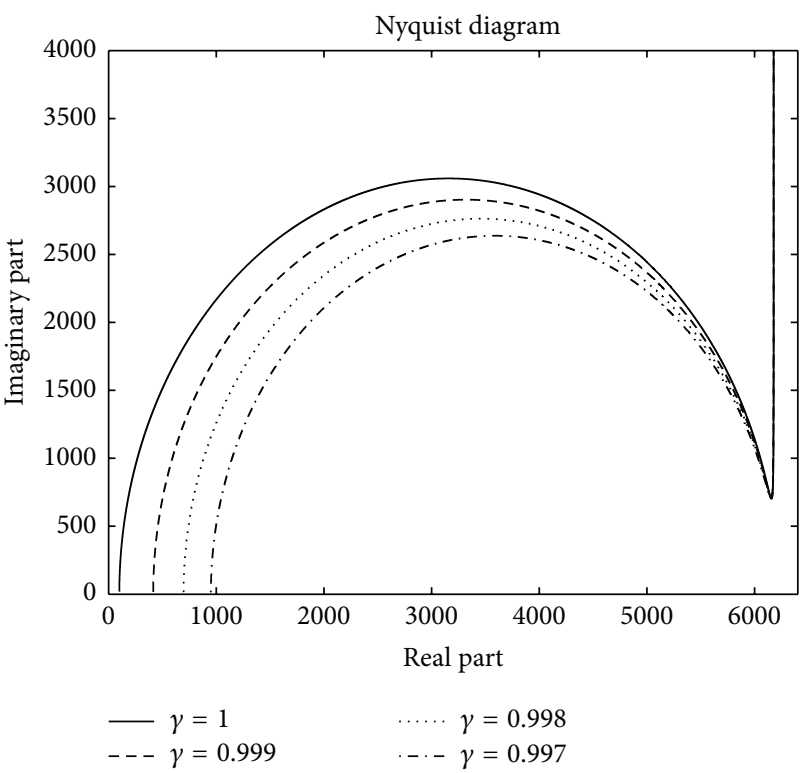

(a)

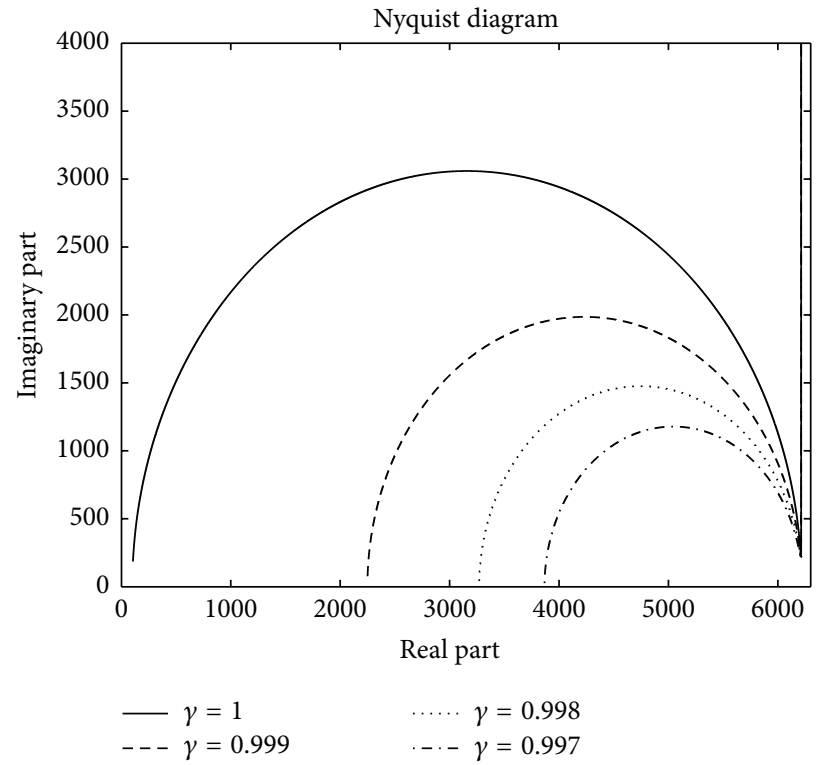

(c)
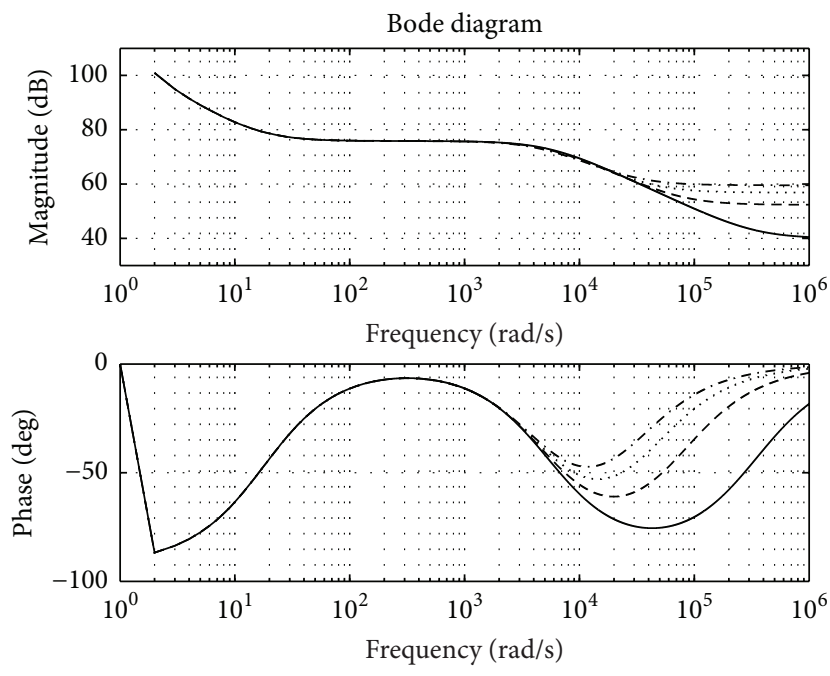

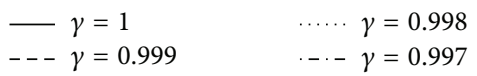

(b)
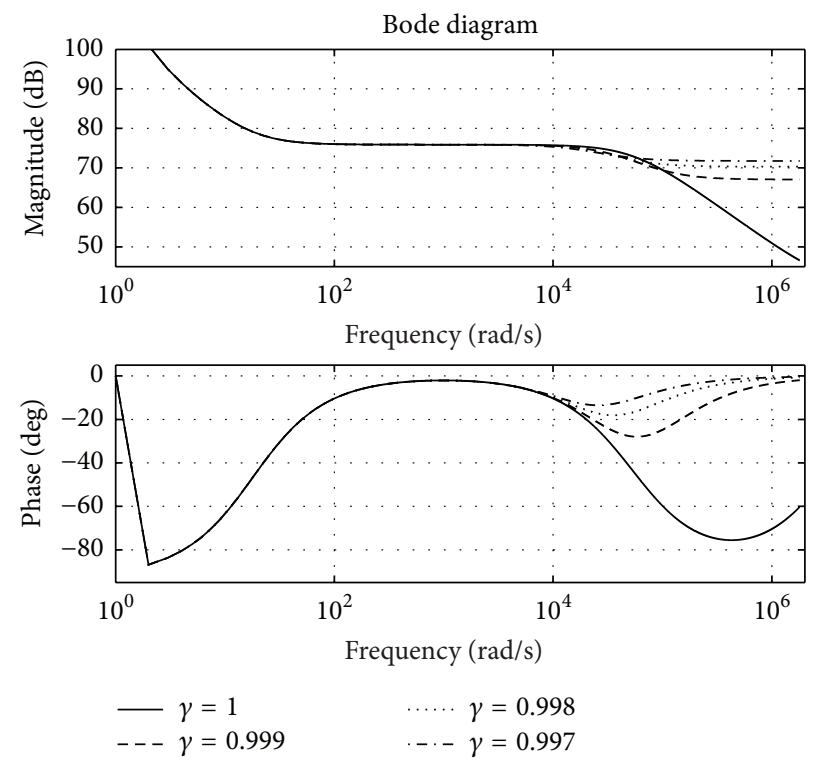

(d)

FIGURE 10: Nyquist and Bode diagram for the limited diffusion model, Caputo-Fabrizio derivative approach, in (a) and (b): $R_{s}=100 \Omega$, $R_{p}=5000 \Omega, C_{s}=3^{-6} \mathrm{~F}$, and $C_{p}=1^{-3} \mathrm{~F}$ for $\beta=1 / 2$ corresponding to $C_{s}, \gamma=1, \gamma=0.95, \gamma=0.9$, and $\gamma=0.85$; for $(\mathrm{c})$ and $(\mathrm{d}), R_{p}=5000 \Omega$, $C_{s}=3^{-7} \mathrm{~F}$, and $C_{p}=1^{-3} \mathrm{~F}$ for $\beta=0.4$ corresponding to $C_{s}, \gamma=1, \gamma=0.95, \gamma=0.9$, and $\gamma=0.85$.

elements whose electrochemical properties and structural features or the physicochemical processes are taking place in the studied being. The resulting spectrum described in a wide frequency range might present one, two, or more time constants, depending on the monolayer or multilayer structure, porosity, or diffusive limitations caused by the charge transfer process. The existence of a time constant indicates a homogeneous layer structure; if two different constants are presented, this may indicate the existence of two sublayers.
In the field of biomaterials, the electrochemical impedance spectroscopy technique proves to be useful in characterizing roughness or heterogeneous surfaces, in coatings analysis, as cell suspensions, in studying the adsorption of protein, and in characterizing the performance of materials, mainly with regard to electrocatalysis and corrosion. In this context, FC allows the investigation of the nonlocal response of electrochemical systems. FC has been used successfully to modify many existing models of physical processes; the representation of equivalent models in integer-order 
derivatives provided a good approximation of the electrochemical response of the model.

On the basis of Cole's proposal to add an extra degree of freedom in order to solve the $\mathrm{RC}$ circuits for characterization purposes and improve the correlation in the adjustment to experimental data, we have developed analytical arguments to derive this result based on integration in weighted individual relaxation processes. However, the distributions of relaxation times involve complex functions and are difficult to measure. This study has shown a pattern in which the Cole type behavior appears as a result of competition between a capacitive and resistive behavior within the sample, characterized by the fractional order derivative of the applied voltage. This combination of stored and dissipated energy is conveniently based on the representation of linear viscoelastic behavior; this dissipation is known as internal friction. In the literature it is common to characterize based on least-squares fit of equivalent electrical circuit models on experimental data, including Cole models. From the description of the fractional differential equation models it can be noted that the representation of Cole models is derived as a particular solution to the RC circuit under FC.

Since some authors replace the derivative of a fractional entire order in a purely mathematical context, the physical parameters involved in the differential equation do not have the dimensionality obtained in the laboratory and from the physical point of view of engineering this is not entirely correct. In this representation an auxiliary parameter $\alpha$ is introduced; this parameter characterizes the existence of the fractional temporal components and related the time constant of the system; the solutions presented here preserve the dimensionality of the studied system for any value of the exponent of the fractional derivative. The advantage of this alternative representation in when comparison with the models presented in the literature is the physical compatibility of the solutions.

The Caputo representation has the disadvantage that their kernel had singularity; this kernel includes memory effects and therefore this definition cannot accurately describe the full effect of the memory. Due to this inconvenience, Caputo and Fabrizio in [15] present a new definition of fractional derivative without singular kernel, the Caputo-Fabrizio fractional derivative. The two definitions of fractional derivatives must apply conveniently depending on the nature of the system and the choice of the fractional derivative depends upon the problem studied and on the phenomenological behavior of the system.

\section{Competing Interests}

The authors declare that they have no competing interests.

\section{Acknowledgments}

The authors would like to thank Mayra Martínez for the interesting discussions. J. F. Gómez-Aguilar acknowledges the support provided by CONACYT: catedras CONACYT para jovenes investigadores 2014 .

\section{References}

[1] E. Barsoukov and J. R. Macdonald, Impedance Spectroscopy, Theory, Experiment, and Applications, John Wiley \& Sons, New York, NY, USA, 2005.

[2] K. B. Oldham and J. Spanier, The Fractional Calculus, Academic Press, New York, NY, USA, 1974.

[3] K. S. Miller and B. Ross, An Introduction to the Fractional Calculus and Fractional Differential Equations, John Wiley \& Sons, New York, NY, USA, 1993.

[4] I. Podlubny, Fractional Differential Equations, Academic Press, New York, NY, USA, 1999.

[5] D. Baleanu, K. Diethelm, E. Scalas, and J. J. Trujillo, Fractional Calculus: Models and Numerical Methods, Series on Complexity, Nonlinearity and Chaos, World Scientific, River Edge, NJ, USA, 2012.

[6] R. Hilfer, Applications of Fractional Calculus in Physics, World Scientific, Singapore, 2000.

[7] J. F. Gómez Aguilar and D. Baleanu, "Solutions of the telegraph equations using a fractional calculus approach," Proceedings of the Romanian Academy-Series A, vol. 15, no. 1, pp. 27-34, 2014.

[8] R. L. Magin, Fractional Calculus in Bioengineering, Begell House, Danbury, Conn, USA, 2006.

[9] J. F. Gómez-Aguilar, H. Yépez-Martínez, R. F. Escobar-Jiménez, C. M. Astorga-Zaragoza, L. J. Morales-Mendoza, and M. González-Lee, "Universal character of the fractional spacetime electromagnetic waves in dielectric media," Journal of Electromagnetic Waves and Applications, vol. 29, no. 6, pp. 727740, 2015.

[10] D. A. Benson, M. M. Meerschaert, and J. Revielle, "Fractional calculus in hydrologic modeling: a numerical perspective," Advances in Water Resources, vol. 51, pp. 479-497, 2013.

[11] J. F. Gómez-Aguilar and D. Baleanu, "Fractional transmission line with losses," Zeitschrift für Naturforschung A, vol. 69, no. 10-11, pp. 539-546, 2014.

[12] J. F. Gómez-Aguilar, M. Miranda-Hernández, M. G. LópezLópez, V. M. Alvarado-Martínez, and D. Baleanu, "Modeling and simulation of the fractional space-time diffusion equation," Communications in Nonlinear Science and Numerical Simulation, vol. 30, no. 1-3, pp. 115-127, 2016.

[13] V. Uchaikin, Fractional Derivatives for Physicists and Engineers, Springer, New York, NY, USA, 2012.

[14] K. Diethelm, N. J. Ford, A. D. Freed, and Y. Luchko, "Algorithms for the fractional calculus: a selection of numerical methods," Computer Methods in Applied Mechanics and Engineering, vol. 194, no. 6-8, pp. 743-773, 2005.

[15] M. Caputo and M. Fabrizio, "A new definition of fractional derivative without singular kernel," Progress in Fractional Differentiation and Applications, vol. 1, no. 2, pp. 73-85, 2015.

[16] J. Lozada and J. J. Nieto, "Properties of a new fractional derivative without singular kernel," Progress in Fractional Differentiation and Applications, vol. 1, no. 2, pp. 87-92, 2015.

[17] M. E. Orazem and B. Tribollet, Electrochemical Impedance Spectroscopy, vol. 48, John Wiley \& Sons, New York, NY, USA, 2011.

[18] I. S. Jesus, J. A. Tenreiro MacHado, and J. Boaventure Cunha, "Fractional electrical impedances in botanical elements," Journal of Vibration and Control, vol. 14, no. 9-10, pp. 1389-1402, 2008. 
[19] A. S. Elwakil and B. Maundy, "Extracting the Cole-Cole impedance model parameters without direct impedance measurement," Electronics Letters, vol. 46, no. 20, article 1367, 2010.

[20] K. B. Oldham, "Fractional differential equations in electrochemistry," Advances in Engineering Software, vol. 41, no. 1, pp. 9-12, 2010.

[21] R. Martín, J. J. Quintana, A. Ramos, and I. de la Nuez, "Modeling of electrochemical double layer capacitors by means of fractional impedance," Journal of Computational and Nonlinear Dynamics, vol. 3, no. 2, Article ID 021303, 2008.

[22] I. S. Jesus and J. A. T. Machado, "Comparing integer and fractional models in some electrical systems," in Proceedings of the 4th IFAC Workshop on Fractional Differentiation and its Applications (FDA '10), Badajoz, Spain, October 2010.

[23] I. S. Jesus and J. A. Tenreiro Machado, "Application of integer and fractional models in electrochemical systems," Mathematical Problems in Engineering, vol. 2012, Article ID 248175, 17 pages, 2012.

[24] J.-B. Jorcin, M. E. Orazem, N. Pébère, and B. Tribollet, "CPE analysis by local electrochemical impedance spectroscopy," Electrochimica Acta, vol. 51, no. 8-9, pp. 1473-1479, 2006.

[25] H. Sheng, Y. Chen, and T. Qiu, "Analysis of biocorrosion electrochemical noise using fractional order signal processing techniques," in Fractional Processes and Fractional-Order Signal Processing, pp. 189-202, Springer, London, UK, 2012.

[26] I. S. Jesus and J. A. T. MacHado, "Development of fractional order capacitors based on electrolyte processes," Nonlinear Dynamics, vol. 56, no. 1-2, pp. 45-55, 2009.

[27] K. S. Cole, "Permeability and impermeability of cell membranes for ions," Cold Spring Harbor Symposia on Quantitative Biology, vol. 8, no. 0, pp. 110-122, 1940.

[28] P. Debye, Polar Molecules, Dover, New York, NY, USA, 1945.

[29] B.-Y. Chang and S.-M. Park, "Electrochemical impedance spectroscopy," Annual Review of Analytical Chemistry, vol. 3, no. 1, pp. 207-229, 2010.

[30] I. Podlubny, "Geometric and physical interpretation of fractional integration and fractional differentiation," Fractional Calculus \&; Applied Analysis, vol. 5, no. 4, pp. 367-386, 2002.

[31] M. Moshrefi-Torbati and J. K. Hammond, "Physical and geometrical interpretation of fractional operators," Journal of the Franklin Institute, vol. 335, no. 6, pp. 1077-1086, 1998.

[32] J. F. Gómez-Aguilara, R. Razo-Hernández, and D. GranadosLieberman, "A physical interpretation of fractional calculus in observables terms: analysis of the fractional time constant and the transitory response," Revista Mexicana de Fisica, vol. 60, no. 1, pp. 32-38, 2014.

[33] R. Hirayama and S. Hanuyama, "Electrochemical impedance for degraded coated steel having pores," Corrosion, vol. 47, no. 12, pp. 952-958, 1991.

[34] A. Amirudin and D. Thieny, "Application of electrochemical impedance spectroscopy to study the degradation of polymercoated metals," Progress in Organic Coatings, vol. 26, no. 1, pp. 1-28, 1995.

[35] P. L. Bonora, F. Deflorian, and L. Fedrizzi, "Electrochemical impedance spectroscopy as a tool for investigating underpaint corrosion," Electrochimica Acta, vol. 41, no. 7-8, pp. 1073-1082, 1996.

[36] F. Deflorian, L. Fedrizzi, S. Rossi, and P. L. Bonora, "Organic coating capacitance measurement by EIS: ideal and actual trends," Electrochimica Acta, vol. 44, no. 24, pp. 4243-4249, 1999.

[37] M. Criado, I. Sobrados, J. M. Bastidas, and J. Sanz, "Steel corrosion in simulated carbonated concrete pore solution its protection using sol-gel coatings," Progress in Organic Coatings, vol. 88, pp. 228-236, 2015.

[38] L. Kong and W. Chen, "Carbon nanotube and graphene-based bioinspired electrochemical actuators," Advanced Materials, vol. 26, no. 7, pp. 1025-1043, 2014.

[39] K. R. Ansari, M. A. Quraishi, and A. Singh, "Schiff's base of pyridyl substituted triazoles as new and effective corrosion inhibitors for mild steel in hydrochloric acid solution," Corrosion Science, vol. 79, pp. 5-15, 2014.

[40] S. Shreepathi, A. K. Guin, S. M. Naik, and M. R. Vattipalli, "Service life prediction of organic coatings: electrochemical impedance spectroscopy vs actual service life," Journal of Coatings Technology Research, vol. 8, no. 2, pp. 191-200, 2011.

[41] F. Cadena, L. Irusta, and M. J. Fernandez-Berridi, "Performance evaluation of alkyd coatings for corrosion protection in urban and industrial environments," Progress in Organic Coatings, vol. 76, no. 9, pp. 1273-1278, 2013.

[42] J. A. Calderón-Gutierrez and F. E. Bedoya-Lora, "Barrier property determination and lifetime prediction by electrochemical impedance spectroscopy of a high performance organic coating," Dyna, vol. 81, no. 183, pp. 97-106, 2014.

[43] L. Gómez, A. Quintero, D. Peña, and H. Estupiñan, “Obtención, caracterización y evaluación in vitro de recubrimientos de policaprolactona-quitosano sobre la aleación Ti6Al4V tratada químicamente," Revista de Metalurgia, vol. 50, no. 3, article e21, 2014.

[44] A. I. Muñoz, J. G. Antón, J. L. Guiñón, and V. P. Herranz, “The effect of chromate in the corrosion behavior of duplex stainless steel in $\mathrm{LiBr}$ solutions," Corrosion Science, vol. 48, no. 12, pp. 4127-4151, 2006.

[45] G. Herting, I. O. Wallinder, and C. Leygraf, "Factors that influence the release of metals from stainless steels exposed to physiological media," Corrosion Science, vol. 48, no. 8, pp. 21202132, 2006.

[46] A. Norlin, J. Pan, and C. Leygraf, "Investigation of interfacial capacitance of $\mathrm{Pt}$, Ti and TiN coated electrodes by electrochemical impedance spectroscopy," Biomolecular Engineering, vol. 19, no. 2-6, pp. 67-71, 2002.

[47] A. Miszczyk and K. Darowicki, "Multispectral impedance quality testing of coil-coating system using principal component analysis," Progress in Organic Coatings, vol. 69, no. 4, pp. 330334, 2010.

[48] C. Y. Wang, A. Q. Zhao, and X. M. Kong, "Entropy and its quantum thermody-namical implication for anomalous spectral systems," Modern Physics Letters B, vol. 26, no. 07, 2012.

[49] H.-G. Kim, S.-H. Ahn, J.-G. Kim, S. J. Park, and K.-R. Lee, "Electrochemical behavior of diamond-like carbon films for biomedical applications," Thin Solid Films, vol. 475, no. 1-2, pp. 291-297, 2005.

[50] X. M. Liu, S. L. Wu, P. K. Chu et al., "Effects of water plasma immersion ion implantation on surface electrochemical behavior of NiTi shape memory alloys in simulated body fluids," Applied Surface Science, vol. 253, no. 6, pp. 3154-3159, 2007.

[51] G. J. Wan, N. Huang, Y. X. Leng et al., "TiN and Ti-O/TiN films fabricated by PIII-D for enhancement of corrosion and wear resistance of Ti-6Al-4V," Surface and Coatings Technology, vol. 186, no. 1-2, pp. 136-140, 2004. 
[52] E. Katz and I. Willner, "Probing biomolecular interactions at conductive and semiconductive surfaces by impedance spectroscopy: routes to impedimetric immunosensors, DNAsensors, and enzyme biosensors," Electroanalysis, vol. 15, no. 11, pp. 913-947, 2003.

[53] S. Erakovic, A. Jankovic, G. C. P. Tsui, C.-Y. Tang, V. MiskovicStankovic, and T. Stevanovic, "Novel bioactive antimicrobial lignin containing coatings on titanium obtained by electrophoretic deposition," International Journal of Molecular Sciences, vol. 15, no. 7, pp. 12294-12322, 2014. 


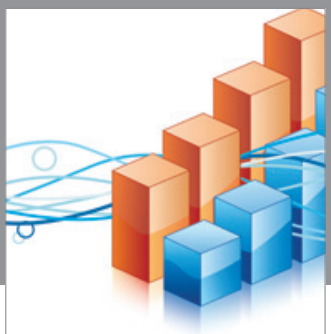

Advances in

Operations Research

vatem alat4

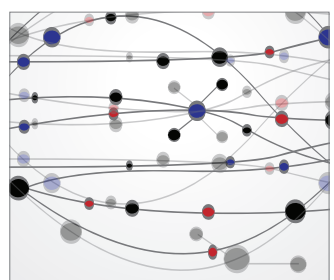

\section{The Scientific} World Journal
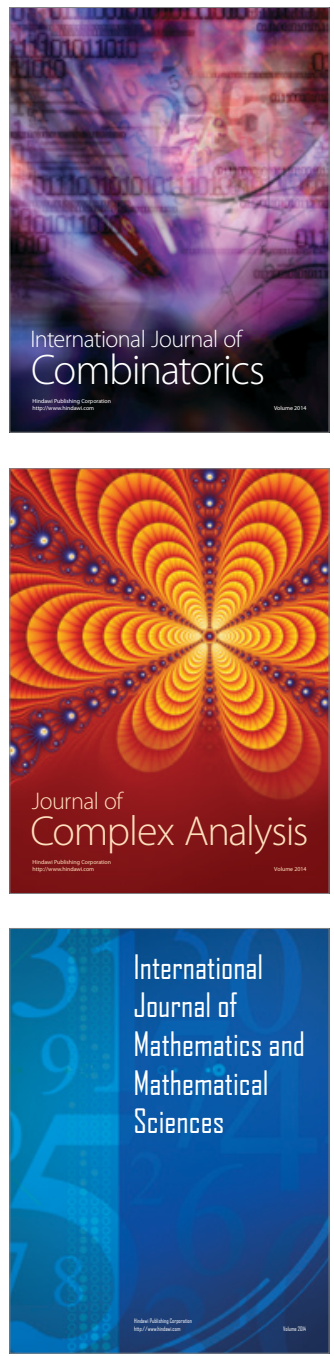
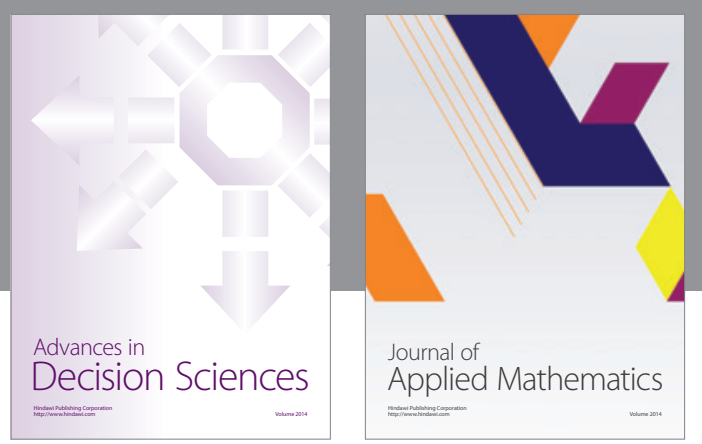

Algebra

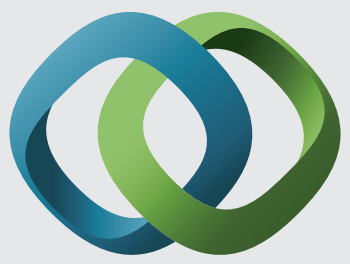

\section{Hindawi}

Submit your manuscripts at

http://www.hindawi.com
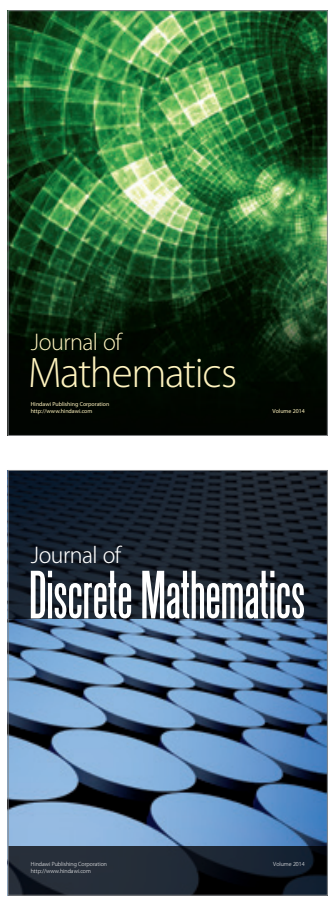

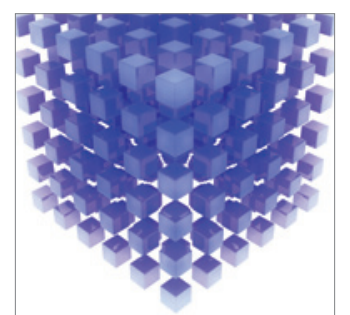

Mathematical Problems in Engineering
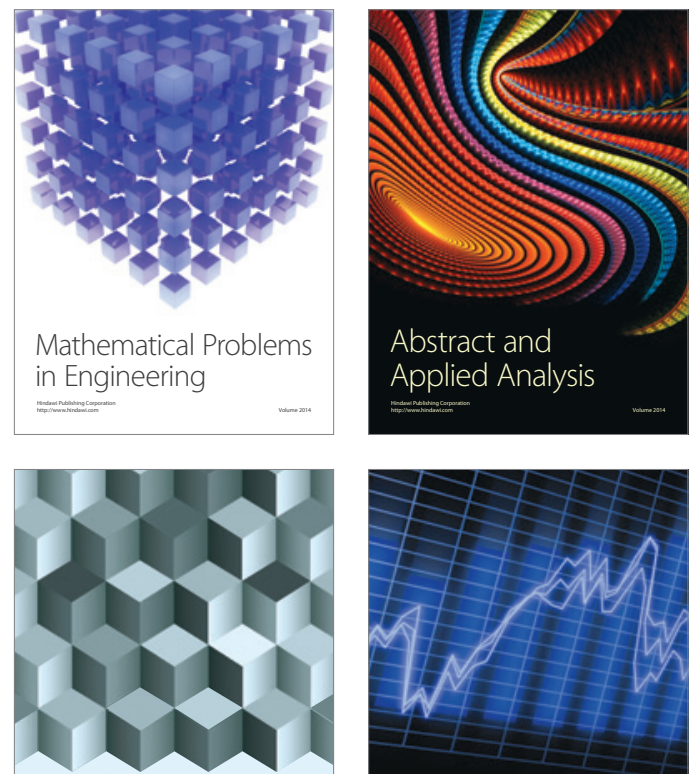

Journal of

Function Spaces

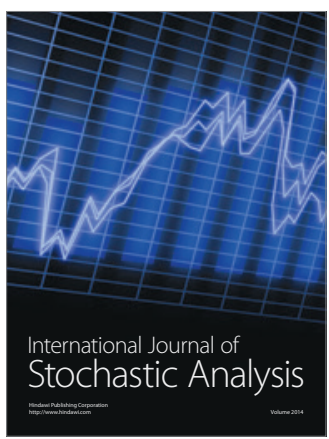

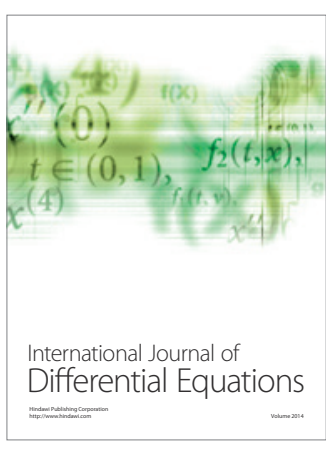
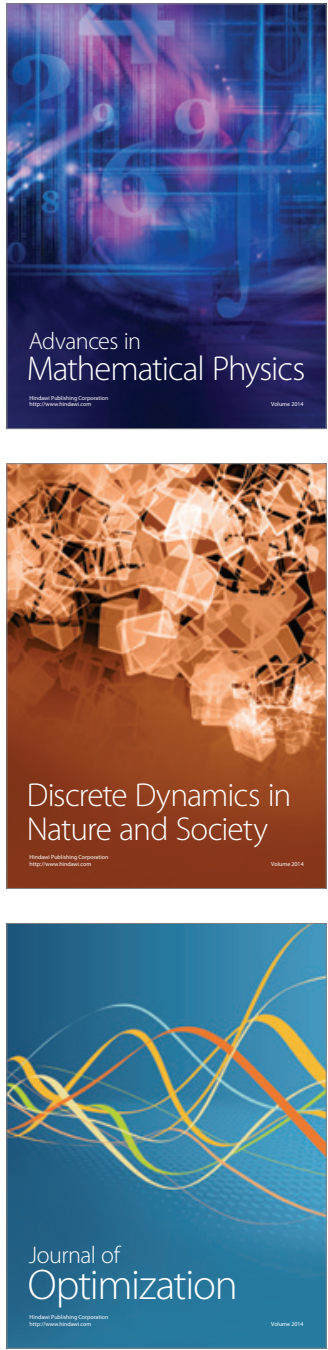